\title{
Real time lattice gauge theory actions
}

\section{Michael Wagman}

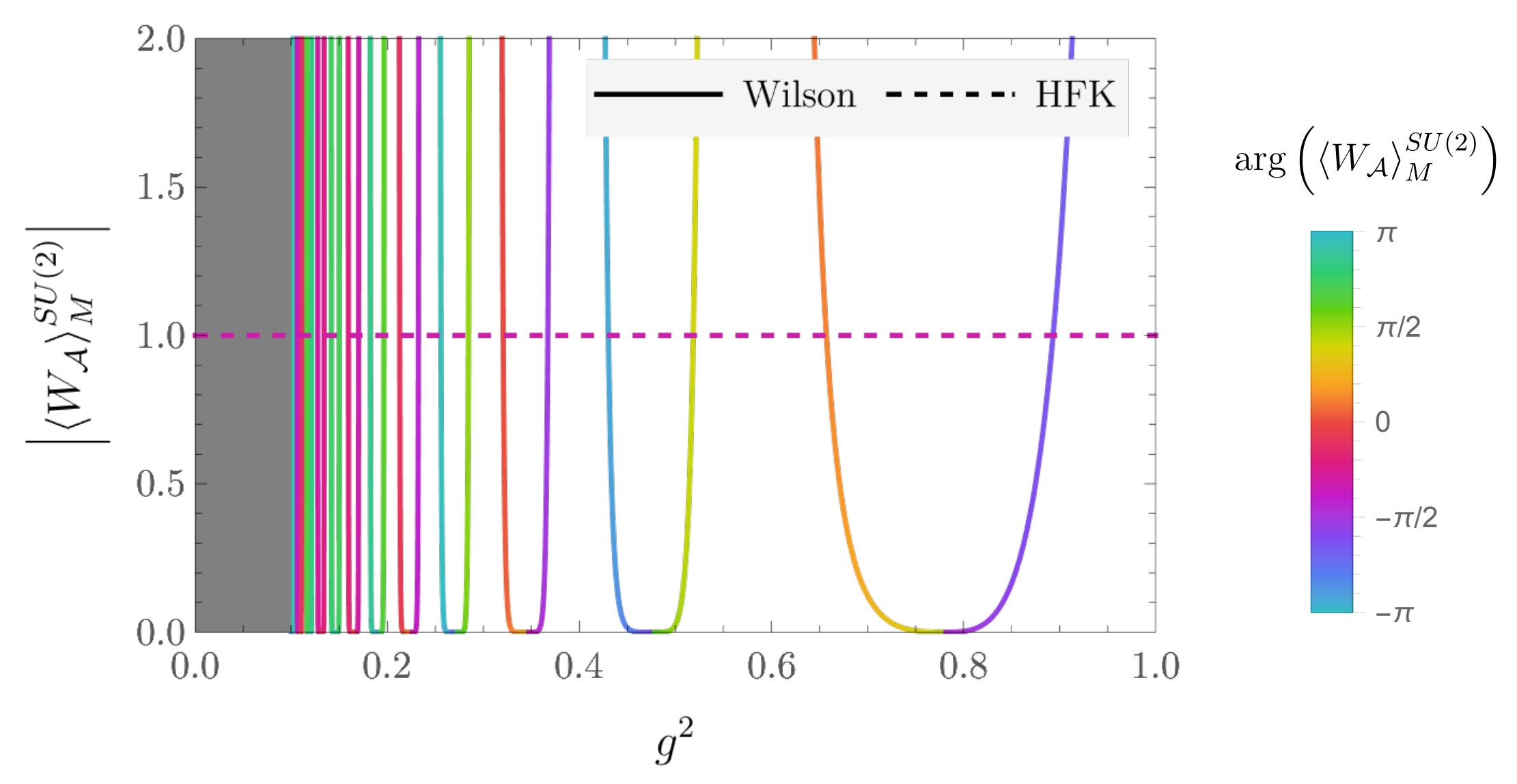

Kanwar, MW, arXiv:2103.02602

MIT faculty lunch

March 4, 2021 


\section{Real-time scattering experiments}

Interesting hadron-hadron and hadron-lepton colliders abound

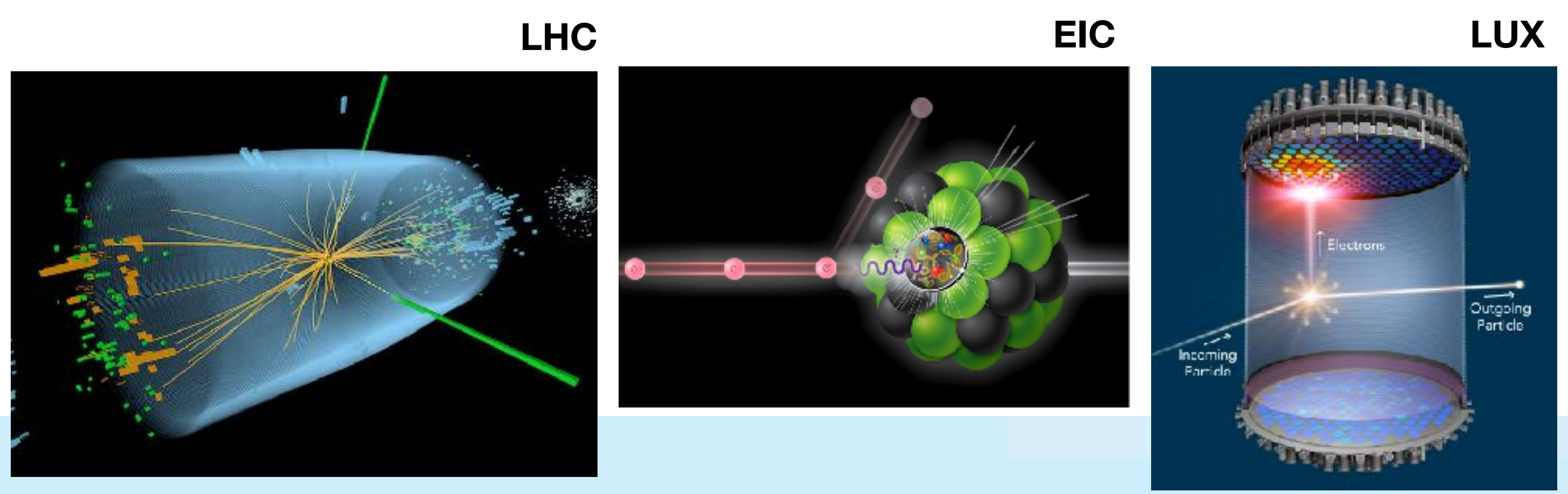

Santord

Underground

Research

Facility

Facility

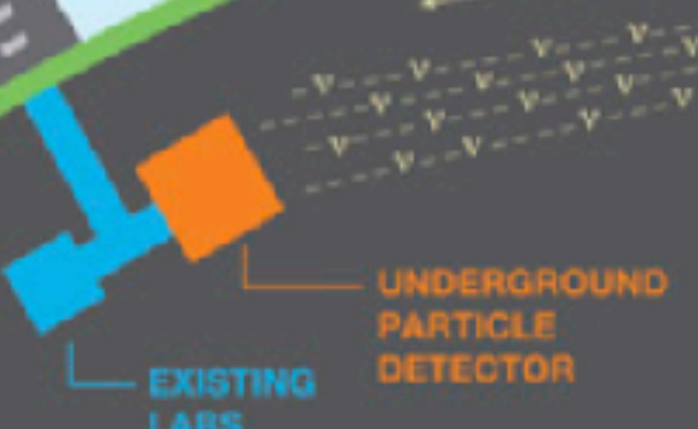

DUNE

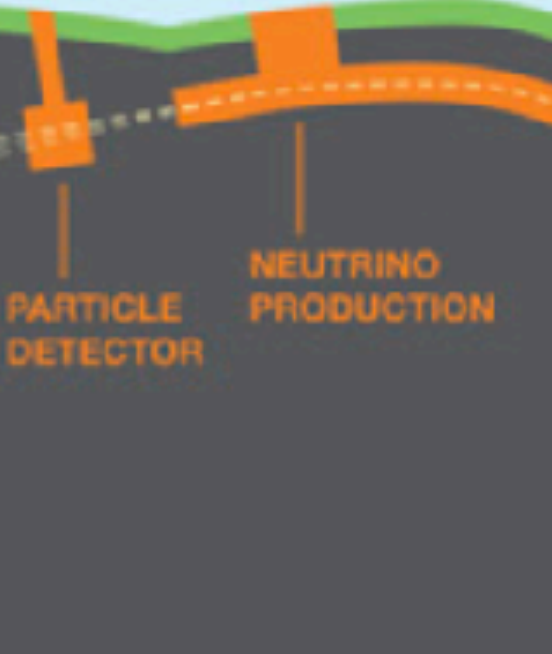




\section{Real-time scattering theory}

Lepton-hadron cross-sections can be predicted using QCD + electroweak perturbation theory
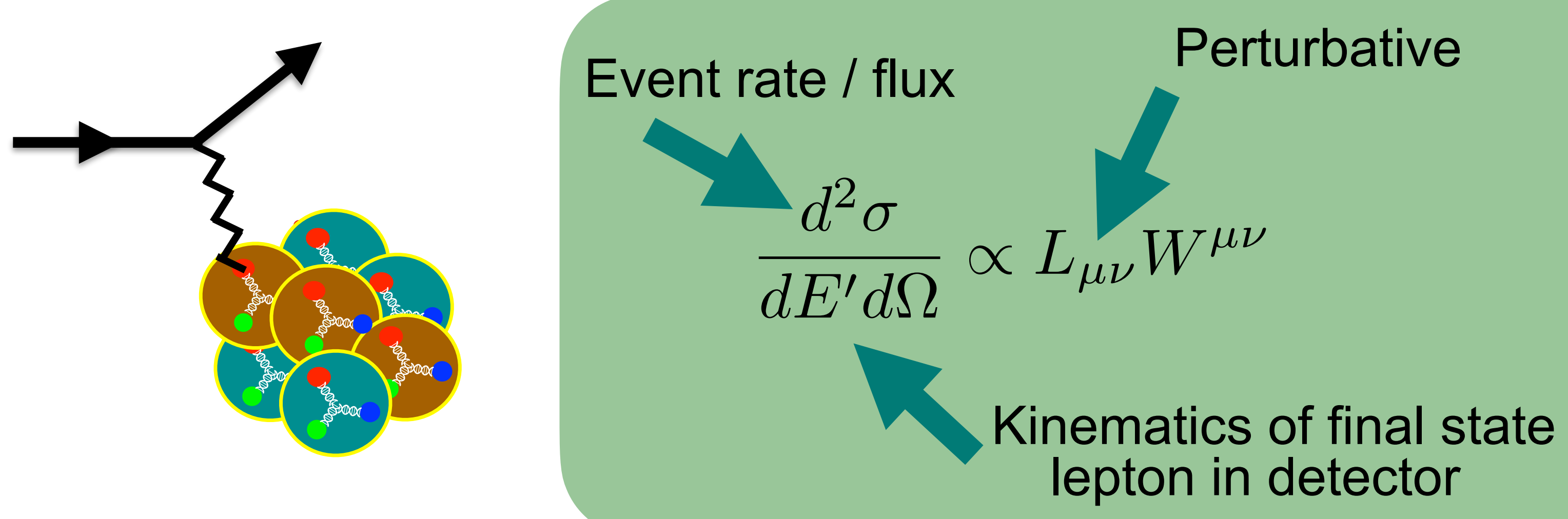

$W^{\mu \nu}=\left\langle f\left|J^{\mu}\left(x^{0}, \vec{x}\right) J^{\nu}\left(y^{0}, \vec{y}\right)\right| i\right\rangle \quad$ All the QCD stuff 


\section{It's hard to imagine}
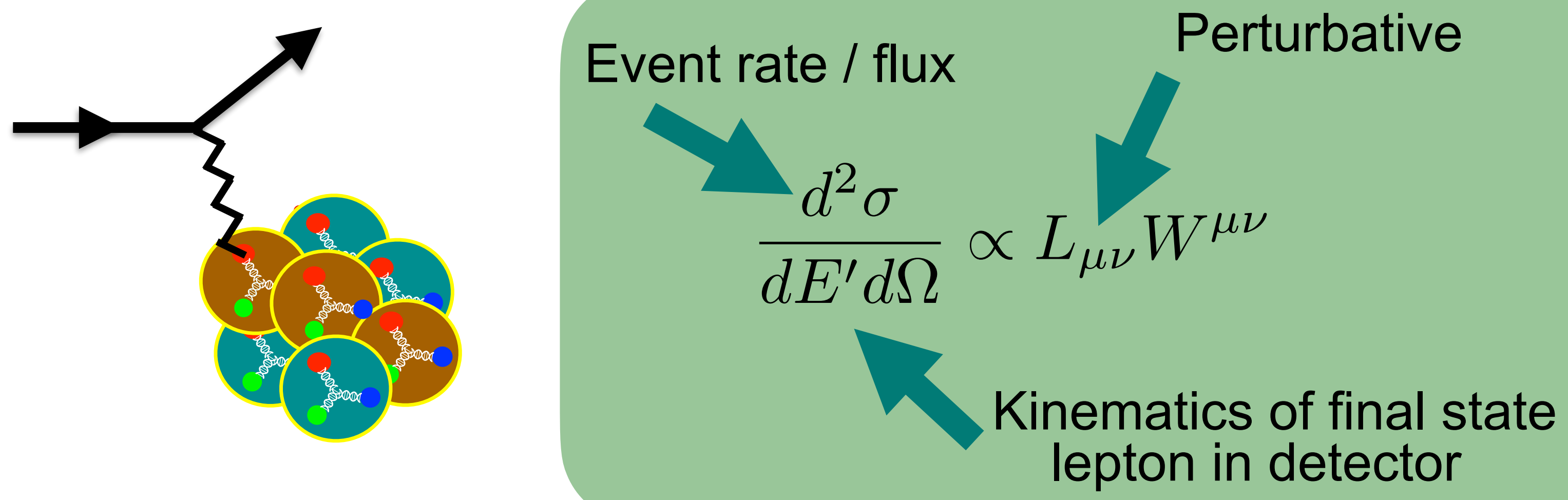

$$
\begin{aligned}
W^{\mu \nu} & =\left\langle f\left|J^{\mu}\left(x^{0}, \vec{x}\right) J^{\nu}\left(y^{0}, \vec{y}\right)\right| i\right\rangle \\
& =\sum_{n} e^{-i E_{n}\left(x^{0}-y^{0}\right)} \rho^{\mu \nu}\left(E_{n}\right) \neq \sum_{n} e^{-E_{n}\left(x^{0}-y^{0}\right)} \rho^{\mu \nu}\left(E_{n}\right)
\end{aligned}
$$

Real-time hadron tensor not simply related to imaginary time version 


\section{Lattice QCD and $\nu A$}

LQCD can provide accurate constraints on $\nu A$ cross sections at a wide range of energies with complementary strengths and weaknesses to experiment

See USQCD $\nu A$ white paper: Kronfeld et al Eur. Phys. J. A 55 (2019)
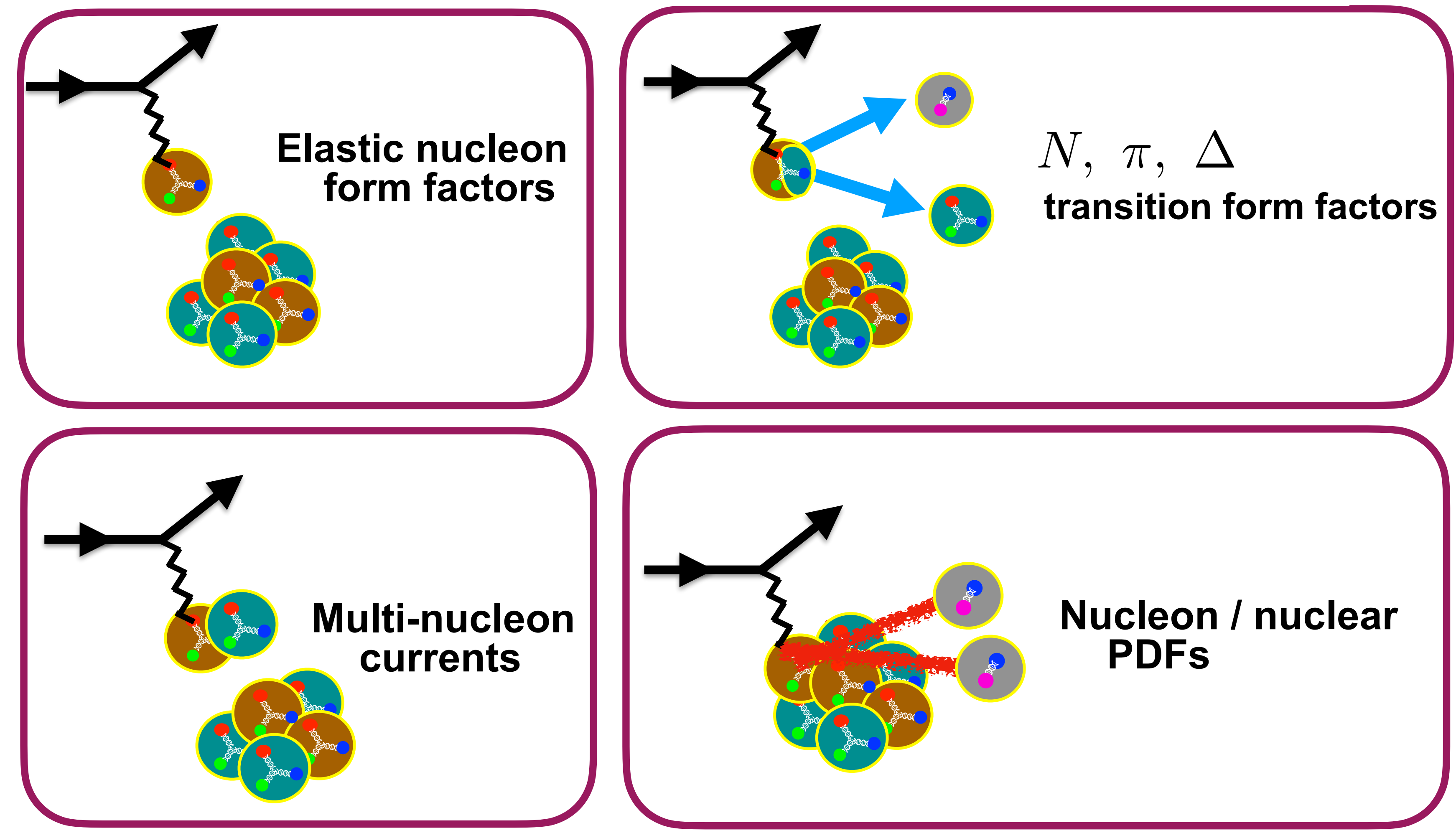


\section{Orders of limits}

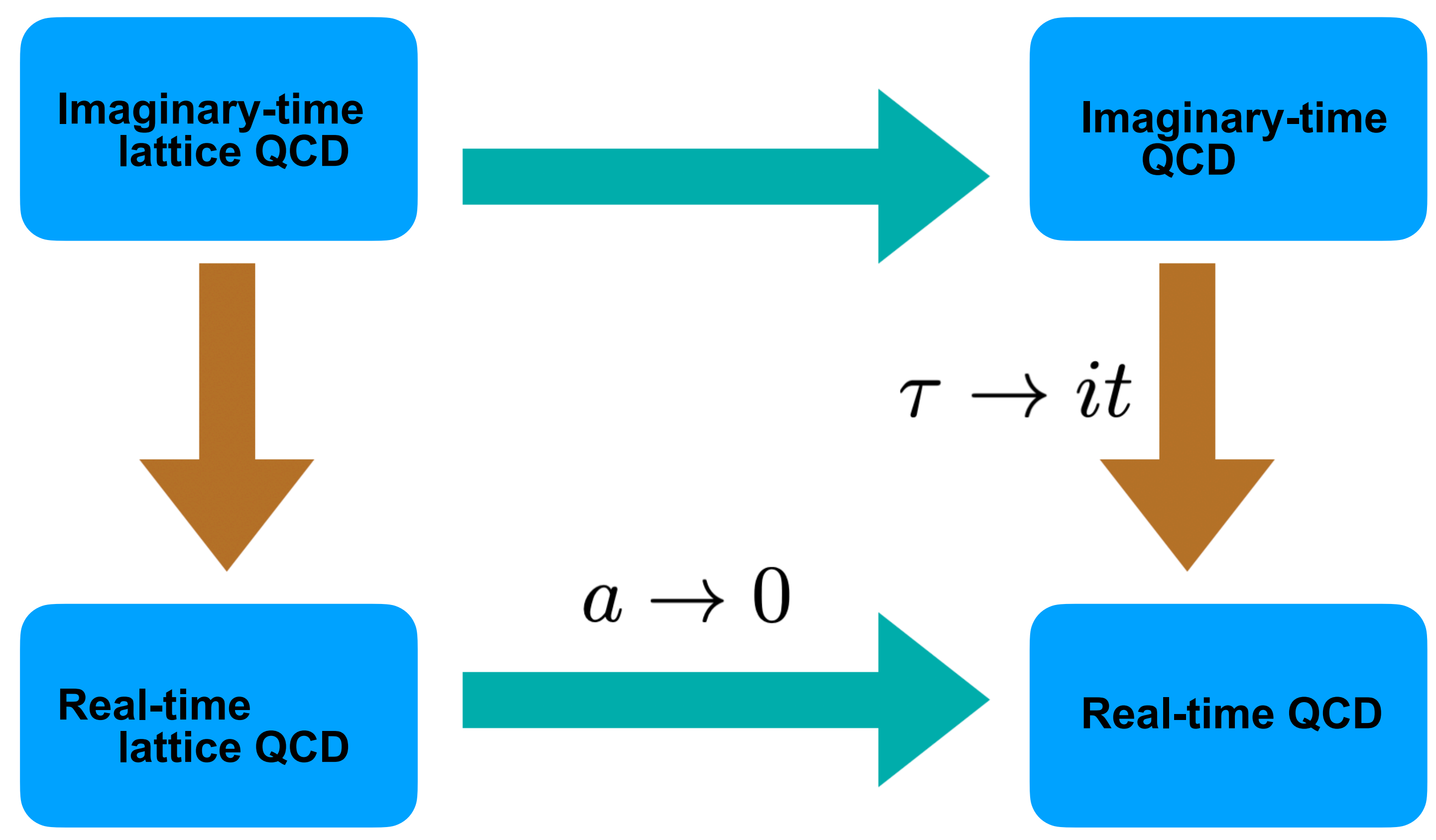




\section{Orders of limits}

Usual lattice QCD strategy

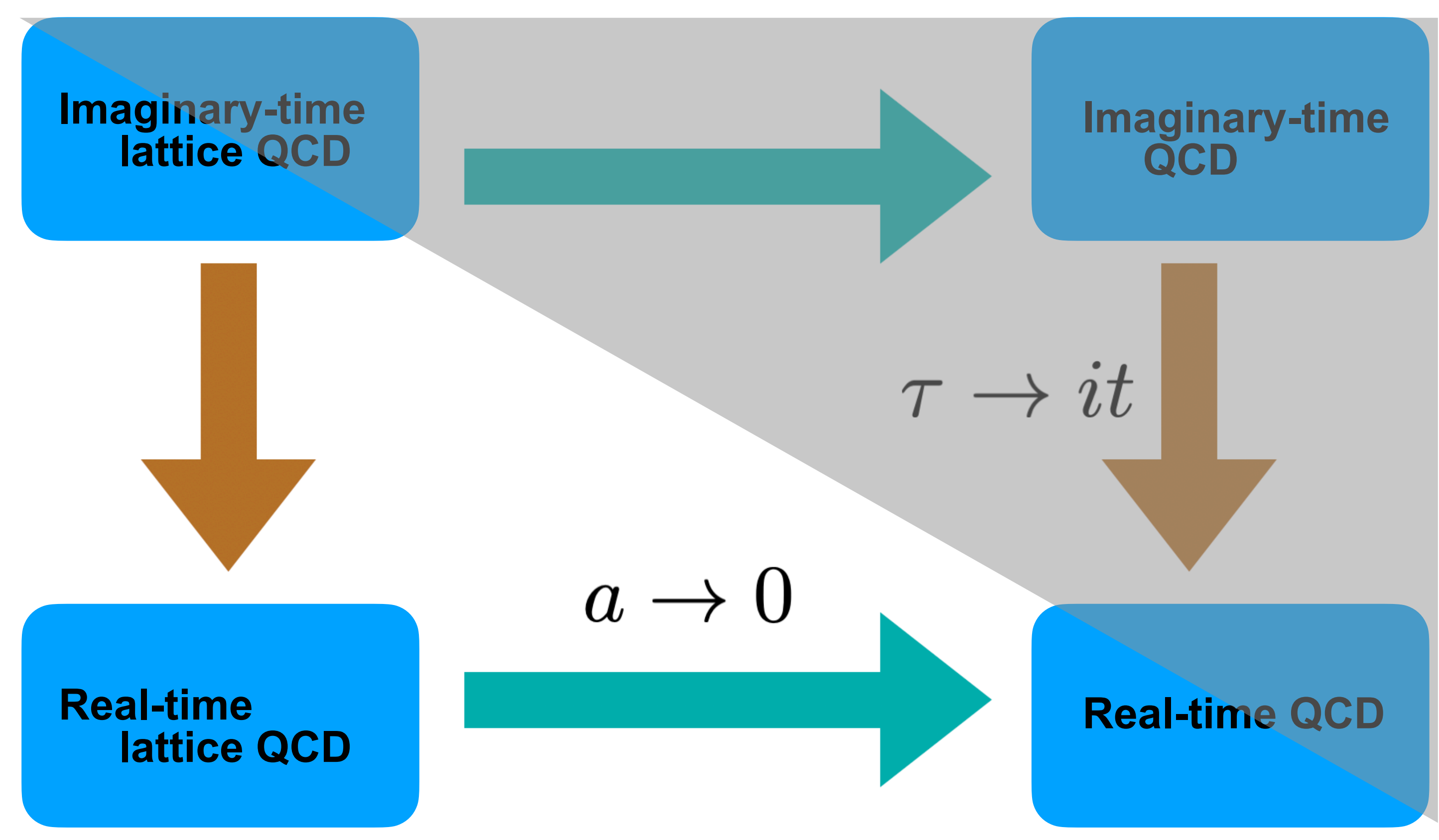




\section{Orders of limits}

\section{Usual lattice QCD strategy}

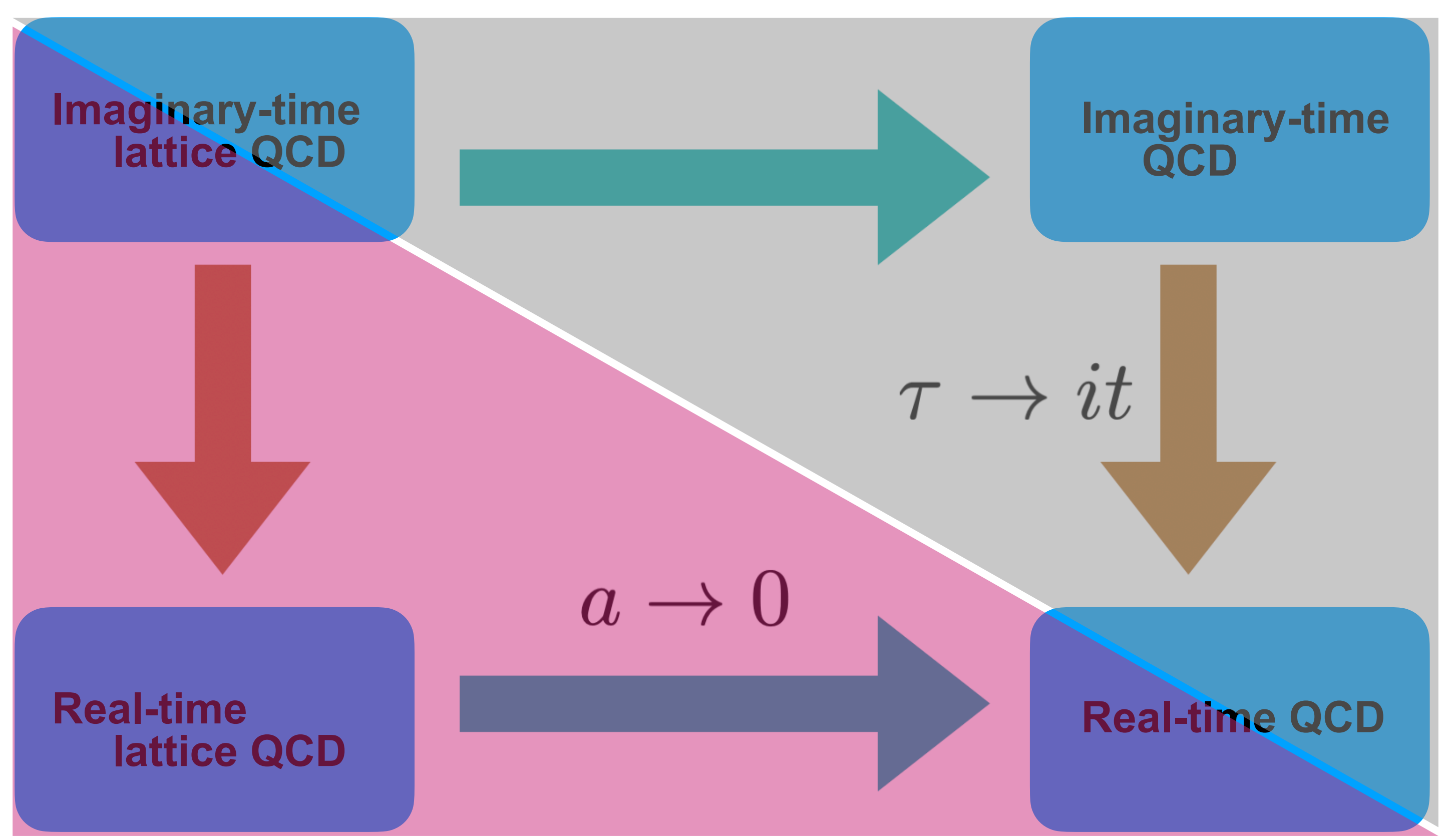

This talk

Signs of trouble first pointed out in Hoshina, Fujii, Kikukawa, PoS LATTICE2019, 190 (2020) 


\section{The Simple Harmonic Oscillator}

Continuum SHO action:

$$
S_{M}[x(t)]=\int d t \frac{1}{2}\left(\partial_{t} x(t)\right)^{2}-\frac{\omega^{2}}{2} x(t)^{2}
$$

\section{Real-time}

Path integral definition:

$$
<x^{\prime}\left|e^{-i \hat{H} L_{T}}\right| x>=\int_{x_{0}=x}^{x_{L_{T}}=x^{\prime}} \mathcal{D} x e^{i S_{M}}
$$

$$
S_{E}[x(t)]=\int d t \frac{1}{2}\left(\partial_{t} x(t)\right)^{2}+\frac{\omega^{2}}{2} x(t)^{2}
$$

\section{Imaginary-time}

$$
<x^{\prime}\left|e^{-\hat{H} L_{T}}\right| x>=\int_{x_{0}=x}^{x_{L_{T}}=x^{\prime}} \mathcal{D} x e^{-S_{E}}
$$




\section{The lattice SHO}

Lattice SHO action

$S_{M}\left(x_{t}\right)=\sum_{n=0}^{L_{T} / a-1} \frac{1}{2 a}\left(x_{n a+a}-x_{n a}\right)^{2}-\frac{\omega^{2}}{2} x_{n a}^{2}$

$S_{E}\left(x_{t}\right)=\sum_{n=0}^{L_{T} / a-1} \frac{1}{2 a}\left(x_{n a+a}-x_{n a}\right)^{2}+\frac{\omega^{2}}{2} x_{n a}^{2}$

Lattice SHO path intgerals

$$
\int_{x_{0}=x}^{x_{L_{T}}=x^{\prime}} \mathcal{D} x e^{i S_{M}}=<x^{\prime}\left|\prod_{n=0}^{L_{T} / a-1} \hat{T}_{M}\right| x>
$$$$
\int_{x_{0}=x}^{x_{L_{T}}=x^{\prime}} \mathcal{D} x e^{-S_{E}}=<x^{\prime}\left|\prod_{n=0}^{L_{T} / a-1} \hat{T}_{E}\right| x>
$$

Transfer matrix:

$$
\left\langle x_{(n+1) a}^{\prime}\left|\hat{T}_{M}\right| x_{n a}\right\rangle=e^{\frac{i}{a \alpha}\left(x_{n a+a}-x_{n a}\right)^{2}-\frac{i \omega^{2}}{2} x_{n a}^{2}}
$$$$
\left\langle x_{(n+1) a}^{\prime}\left|\hat{T}_{E}\right| x_{n a}\right\rangle=e^{-\frac{1}{2 a}\left(x_{n a+a}-x_{n a}\right)^{2}-\frac{\omega^{2}}{2} x_{n a}^{2}}
$$ 


\section{Orders of limits}

For the SHO, the continuum limit commutes with analytic continuation between real and imaginary time

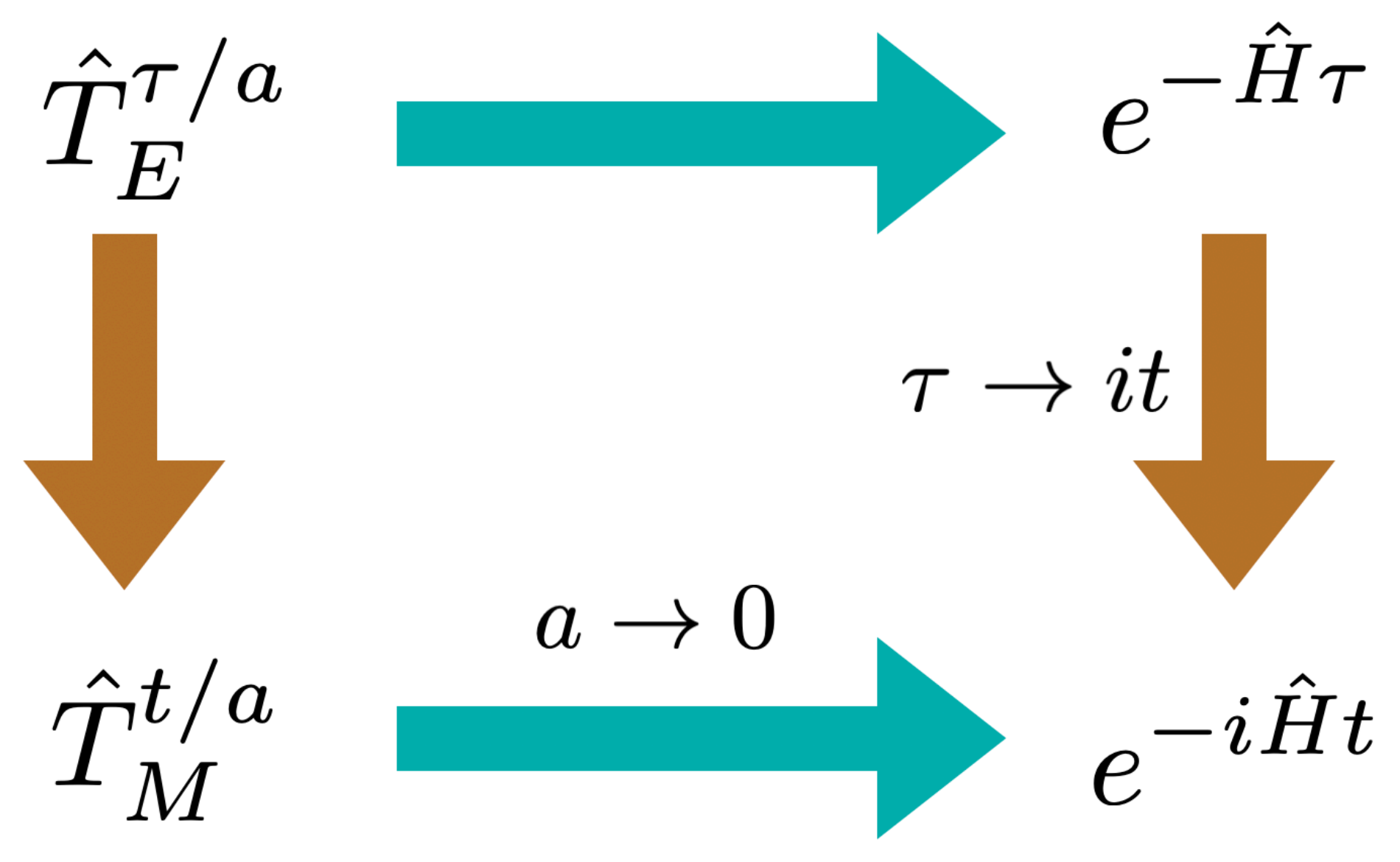

Real-time transfer matrix is unitary

$$
\hat{T}_{M}=e^{-i a \hat{V} / 2} e^{-i a \hat{K}} e^{-i a \hat{V} / 2}
$$

Imaginary-time transfer matrix is positive

$$
\hat{T}_{E}=e^{-a \hat{V} / 2} e^{-a \hat{K}} e^{-a \hat{V} / 2}
$$




\section{The quantum rotator}

Free particle constrained to move on a circle

$$
x(t) \in[0,2 \pi]
$$

Same continuum action can be used as free $\mathrm{SHO}$

Naive discretization breaks periodicity, usual prescription in

Euclidean is to use different action with same small-a behavior

$$
S_{M}\left(x_{t}\right)=\frac{1}{a} \sum_{n=0}^{L_{T} / a-1} 1-\cos \left(x_{n a+a}-x_{n a}\right)
$$

$$
S_{E}\left(x_{t}\right)=\frac{1}{a} \sum_{n=0}^{L_{T} / a-1} 1-\cos \left(x_{n a+a}-x_{n a}\right)
$$

Real-time transfer matrix:

$$
\hat{T}\left(x_{t+a}, x_{t}\right)=e^{\frac{i}{a}-\frac{i}{a} \cos \left(x_{t+a}-x_{t}\right)}
$$




\section{Non-unitarity}

Explicit calculations shows the quantum rotator real-time transfer matrix is non-unitary:

$$
\hat{T}_{M}(x, y) \hat{T}_{M}^{\dagger}\left(y, x^{\prime}\right) \neq \delta\left(x-x^{\prime}\right)
$$

Unitarity requires eigenvalue ratios to have magnitude 1 as $a \rightarrow 0$

But $a \rightarrow 0$ limits of these eigenvalues ratios do not exist for quantum rotators

Real-time

lattice QCD

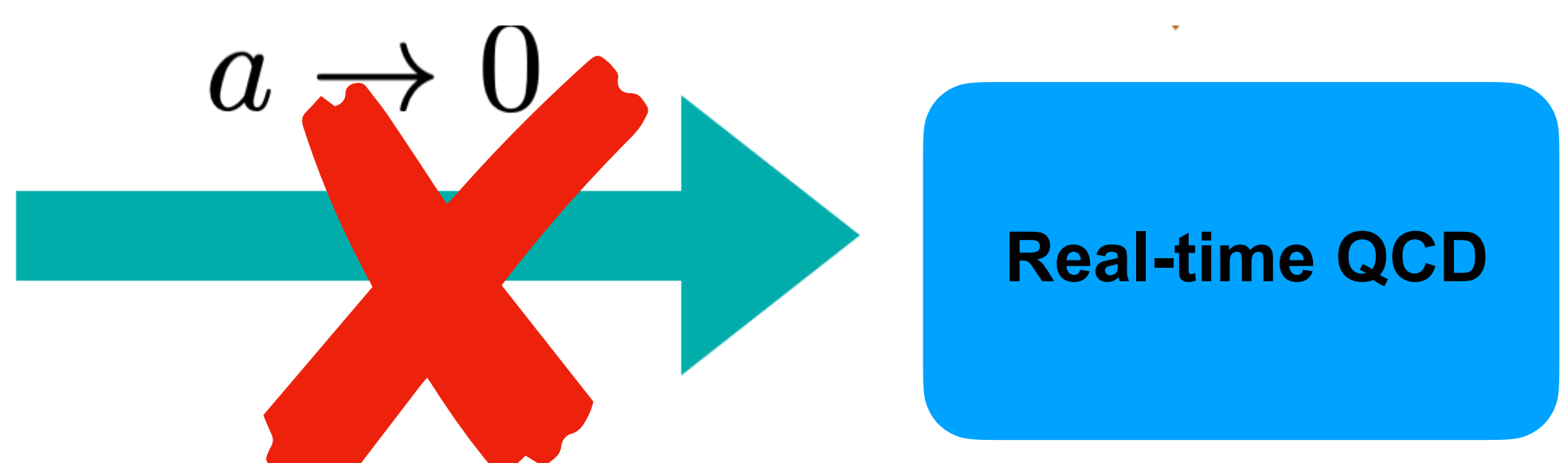




\section{Lattice gauge theory}

Gauge transformations act on matter fields as

$$
\begin{aligned}
& \psi_{x}^{a} \rightarrow \Omega_{x}^{a b} \psi_{x}^{b} \\
& \Omega_{x} \in S U(N), U(1)
\end{aligned}
$$

Gauge field acts as parallel transporter in color space

$$
\begin{aligned}
& D_{\mu}^{a b} \psi_{x}^{b}=U_{x, \mu}^{a b} \psi_{x+\hat{\mu}}^{b}-\psi_{x}^{a} \\
& D_{\mu} \psi_{x}^{a} \rightarrow \Omega_{x}^{a b} D_{\mu} \psi_{x}^{b}
\end{aligned}
$$

$$
\begin{aligned}
& U_{x, \mu} \in S U(N), U(1) \\
& U_{x, \mu}=e^{i a A_{\mu}(x)} \\
& U_{x, \mu} \rightarrow \Omega_{x} U_{x, \mu} \Omega_{x+\hat{\mu}}^{\dagger}
\end{aligned}
$$

Gauge invariant building blocks:
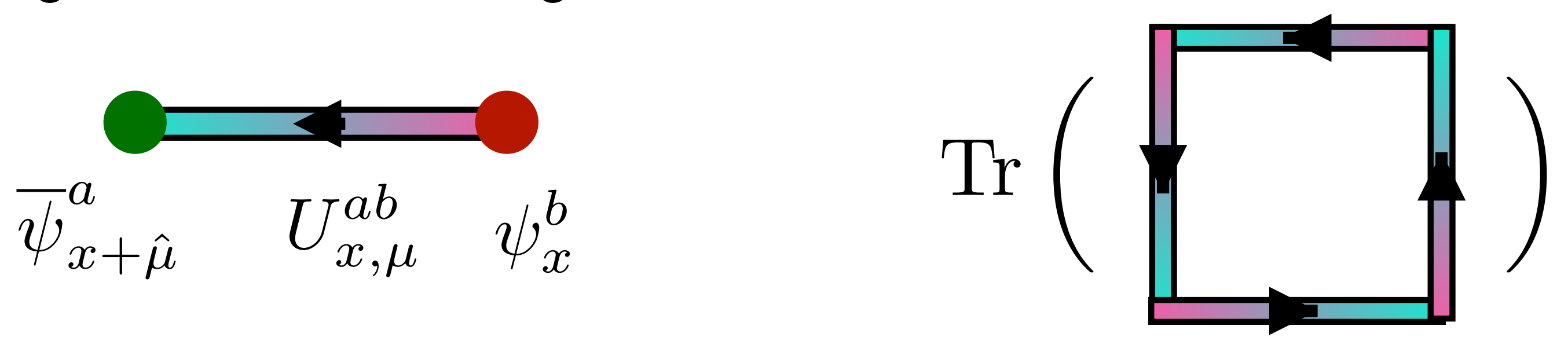


\section{Wilson loops}

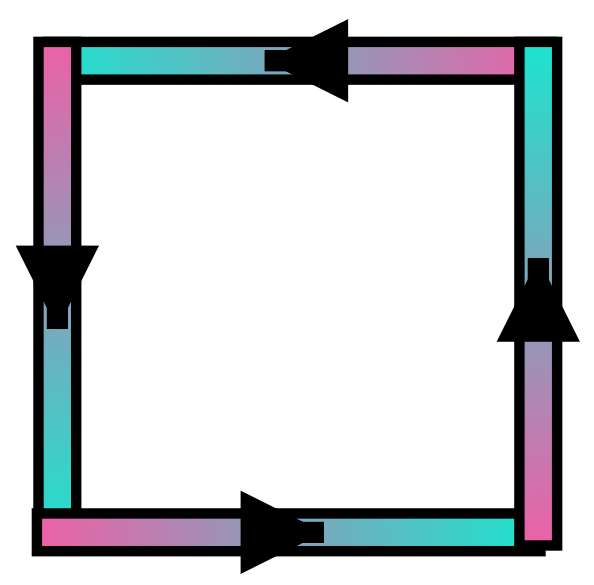

$$
W_{\mathcal{A}}=\prod_{x, \mu \in \partial \mathcal{A}} U_{x, \mu}=
$$
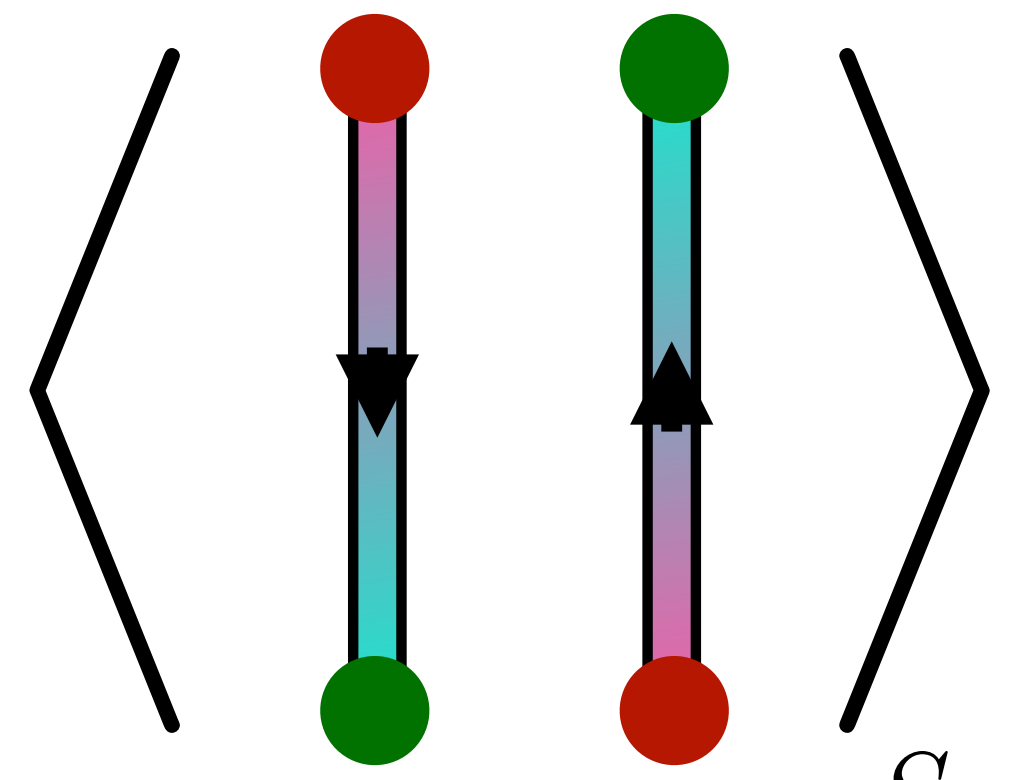

Wilson loops are equivalent to static quark propagators

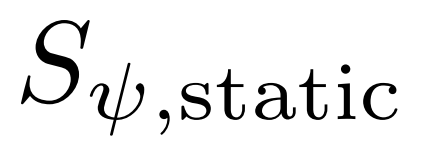

$S_{\psi, \text { static }}=\sum_{x} \bar{\psi}_{x} D_{4} \psi_{x}$

Since by equations of motion $\psi_{(\vec{x}, \tau)}=\prod_{\tau^{\prime}=0}^{\tau} U_{\left(\vec{x}, \tau^{\prime}\right), 4}^{-1} \psi_{(\vec{x}, 0)}$

Static quark potential accessible from Wilson loops

$$
\left\langle W_{r \times \tau}\right\rangle=\sum_{n} Z_{n} e^{-E_{n}(r) \tau}=e^{-V(r) \tau}+\ldots
$$




\section{The Wilson action}

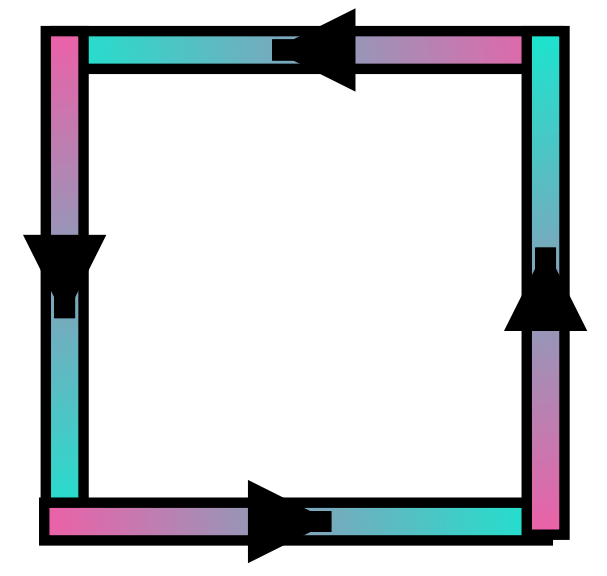

"Plaquettes" are 1x1 Wilson loops

$$
P_{x, \mu \nu}=U_{x, \mu} U_{x+\hat{\mu}, \nu} U_{x+\hat{\mu}+\hat{n u}, \mu}^{-1} U_{x+\hat{\nu}, \nu}^{-1}
$$

Wilson action provides simple, gauge-invariant action with correct naive continuum limit

$$
S_{W}(U)=\frac{1}{g^{2}} \sum_{x} \sum_{\mu<\nu} \operatorname{Tr}\left[2-P_{x, \mu \nu}-P_{x, \mu \nu}^{-1}\right]
$$

Wilson loops can be expressed using plaquettes, most simply taking open boundary conditions and gauge-fixing $U_{x, \nu}=1$

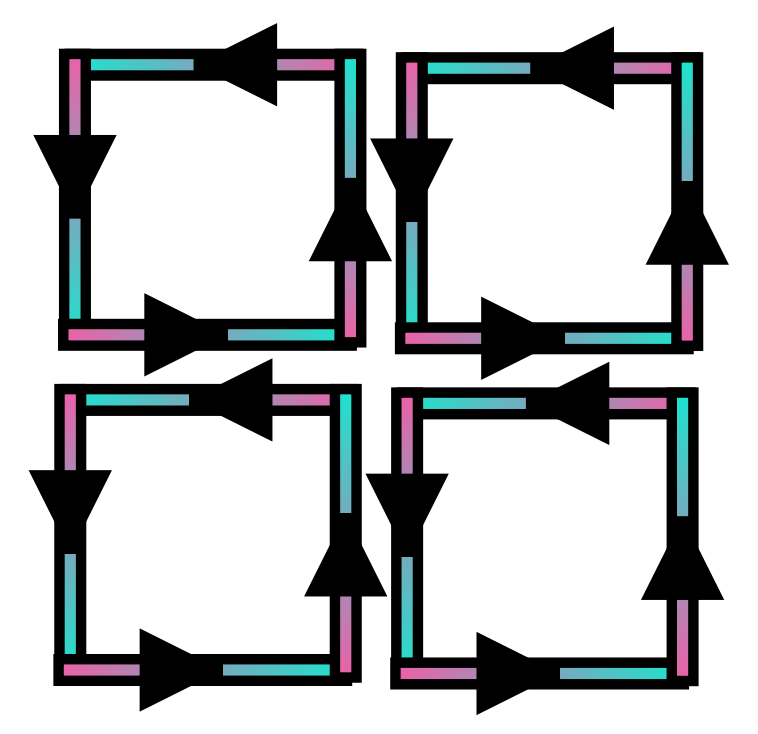

$$
W_{\mathcal{A}}=\prod_{x, \mu \in \partial \mathcal{A}} U_{x, \mu}=\prod_{x \in \mathcal{A}} P_{x, \mu \nu}
$$




\section{D Confinement}

In 2D, Wilson loop expectation values further factorize into products of single-plaquette expectation values

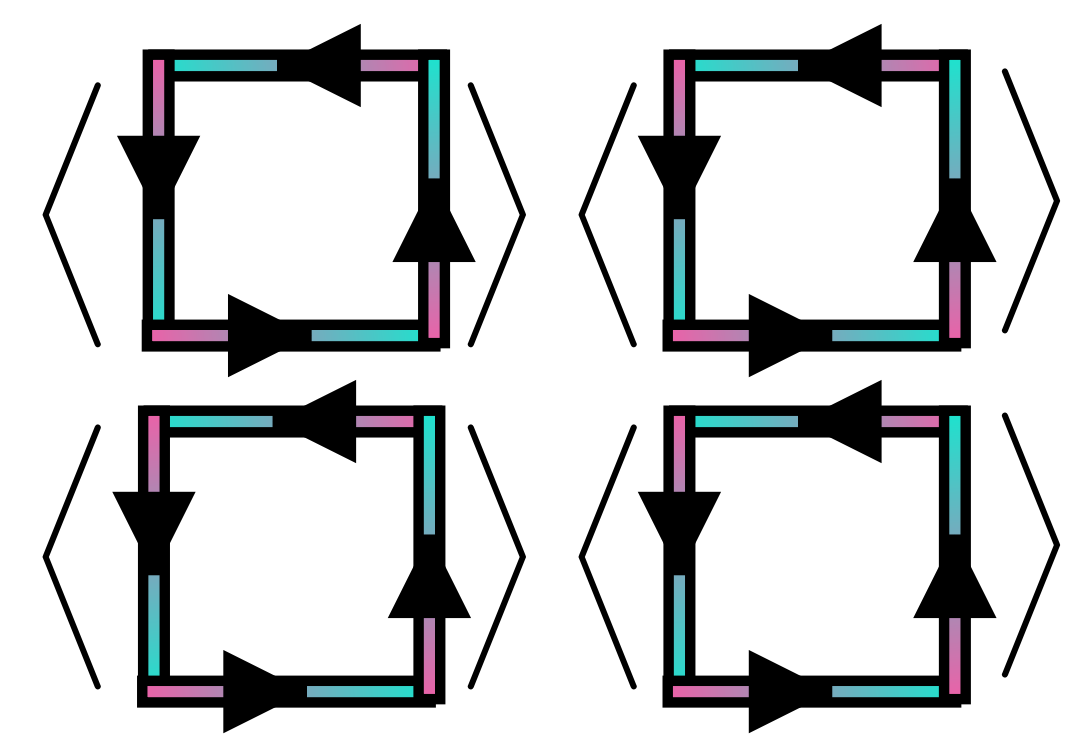

$$
\left\langle\operatorname{Tr}\left(W_{\mathcal{A}}\right)\right\rangle=\prod_{x \in \mathcal{A}}\left\langle\operatorname{Tr}\left(P_{x}\right)\right\rangle=\langle\operatorname{Tr}(P)\rangle^{A}
$$

Implies confinement

$$
\frac{1}{N}\left\langle\operatorname{Tr}\left(W_{\mathcal{A}}\right)\right\rangle=e^{-\sigma A}
$$

Static quark potential $V(r)=\sigma r$

Confining potential arises for any gauge group in 2D from factorization

$$
\begin{gathered}
\sigma_{U(1)}=\ln \left(\frac{I_{0}\left(1 / e^{2}\right)}{I_{1}\left(1 / e^{2}\right)}\right) \\
\sigma_{S U(2)}=\ln \left(\frac{I_{1}\left(4 / g^{2}\right)}{I_{2}\left(4 / g^{2}\right)}\right)
\end{gathered}
$$




\section{The real-time Wilson action}

Wilson action splits into kinetic (timelike plaquettes) and potential (spacelike plaquttes) terms

Making usual sign flips, a real-time Wilson action is obtained

$$
\begin{array}{r}
S_{M, W}(U)=\frac{1}{g^{2}} \sum_{x} \sum_{k} \operatorname{Tr}\left[2-P_{x, 0 k}-P_{x, 0 k}^{-1}\right] \\
-\frac{1}{g^{2}} \sum_{x} \sum_{i<j} \operatorname{Tr}\left[2-P_{x, i j}-P_{x, i j}^{-1}\right]
\end{array}
$$

In (1+1)D only the kinetic term appears, and path integrals are simply related between real- and imaginary-time

$$
e^{i S_{M, W}\left(U, g^{2}\right)}=e^{-S_{E, W}\left(U, i g^{2}\right)}
$$




\section{The Wilson action is non-unitary}

\section{Wick rotate action}

$$
g^{2} \rightarrow i g^{2} \quad\left\langle\frac{1}{2} \operatorname{Tr}\left(W_{\mathcal{A}}\right)\right\rangle_{M, W, S U(2)}=\left[\frac{I_{1}\left(4 i / g^{2}\right)}{I_{2}\left(4 i / g^{2}\right)}\right]^{-L t}
$$

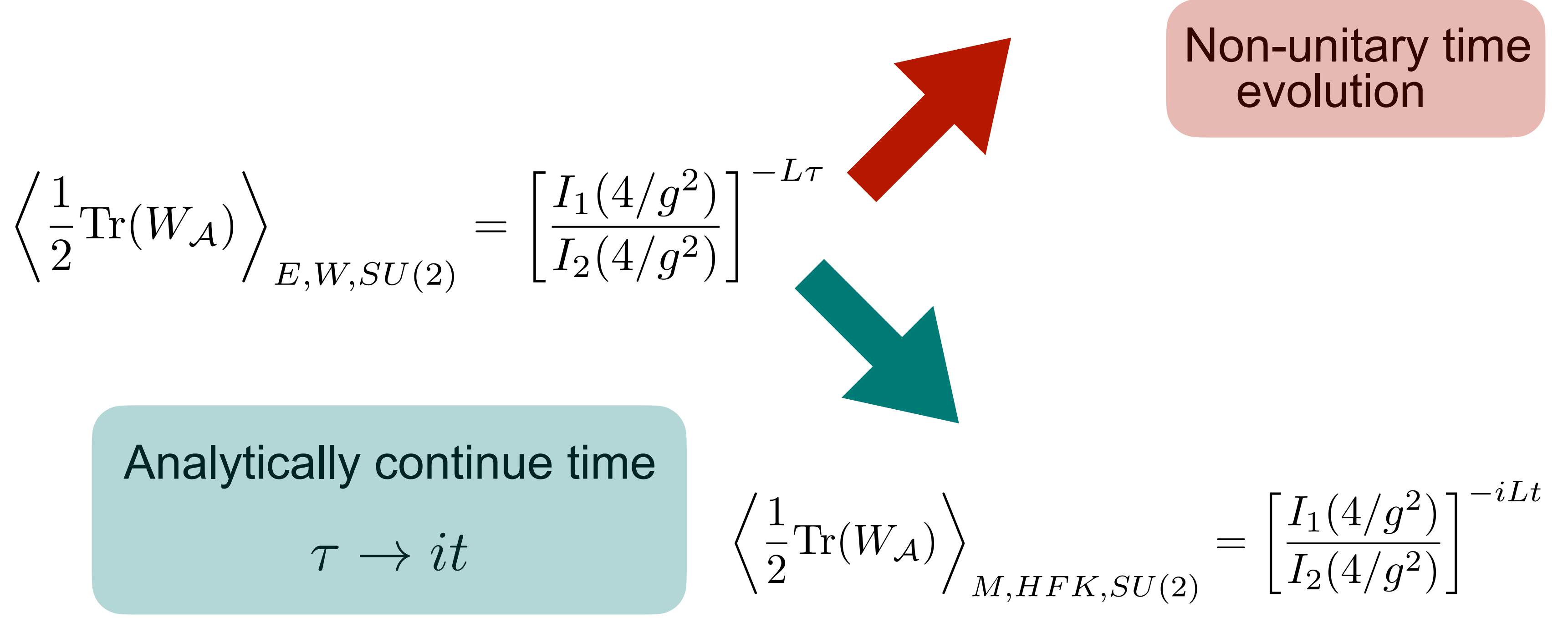




\section{The HFK action}

The non-unitarity of the Wilson real-time transfer matrix was first pointed out by Hoshina, Fujii, and Kikukawa (HFK)

Hoshina, Fujii, Kikukawa, PoS LATTICE2019, 190 (2020)

Starting from the character expansion of the Wilson action

$$
e^{-S_{E, W}(U)}=e^{-\frac{i}{a} V_{W}(U)} \prod_{x, k}\left[\sum_{r} c_{r}^{W}\left(e^{2}\right) \chi_{r}\left(P_{x, 0 k}\right)\right]
$$

The real-time HFK action is defined by replacing the eigenvalues with pure phases to give a unitary transfer matrix by construction

$$
e^{i S_{M, H K F}(U)}=e^{-\frac{i}{a} V_{W}(U)} \prod_{x, k}\left[\sum_{r}\left[c_{r}^{W}\left(e^{2}\right)\right]^{i} \chi_{r}\left(P_{x, 0 k}\right)\right]
$$




\section{Wilson and HFK in (1+1)D}

Analytic calculations using HFK action in (1+1)D recover exact results for analytic continuation of Euclidean Wilson to real time

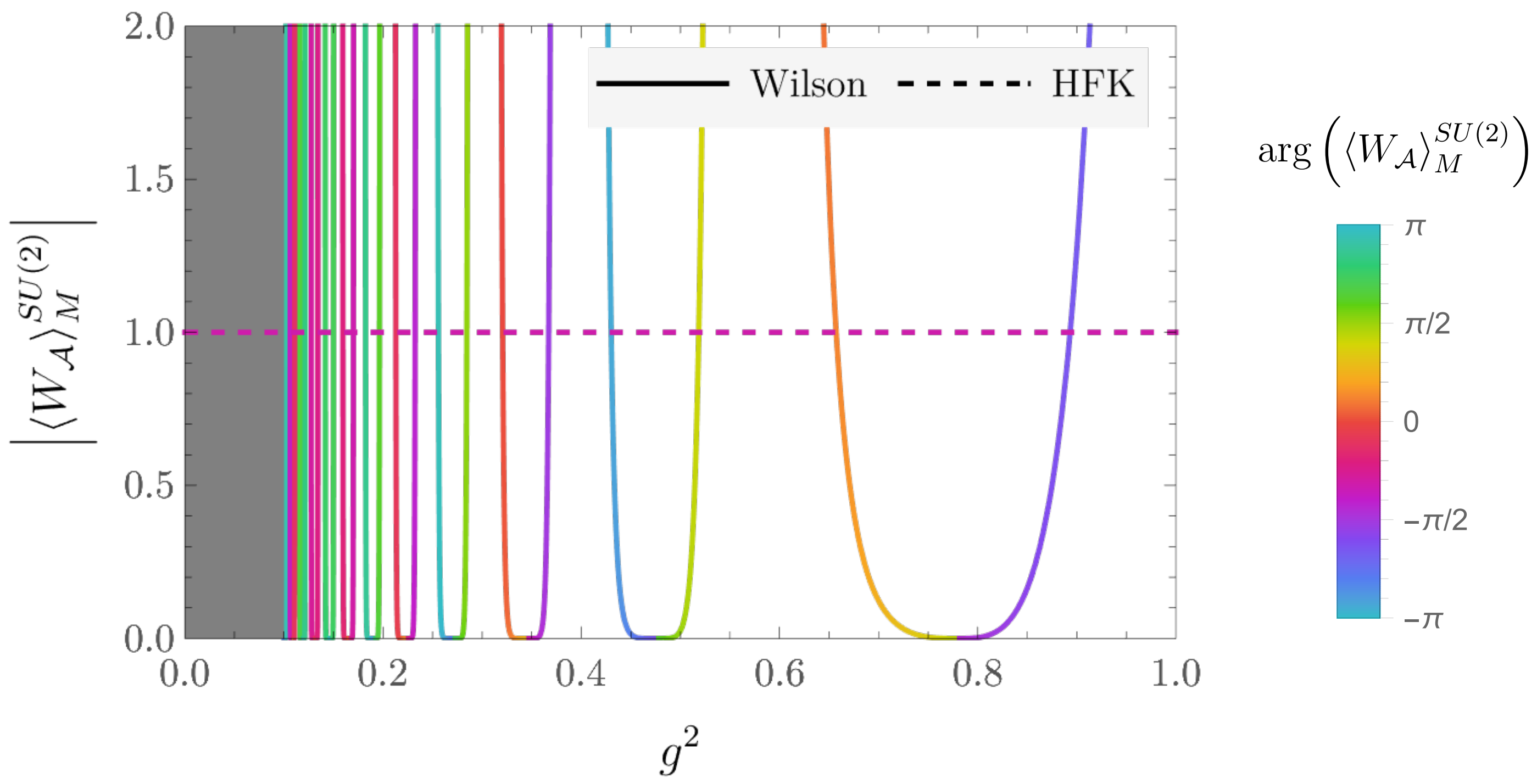

Analytic results for real-time Wilson action show non-unitary continuum limit or have singularities obstructing limit (depends on $\mathrm{N}$ ) 


\section{Divergences}

HFK action well-defined for analytic calculations, but character expansion defining HFK action is a divergent function of gauge field

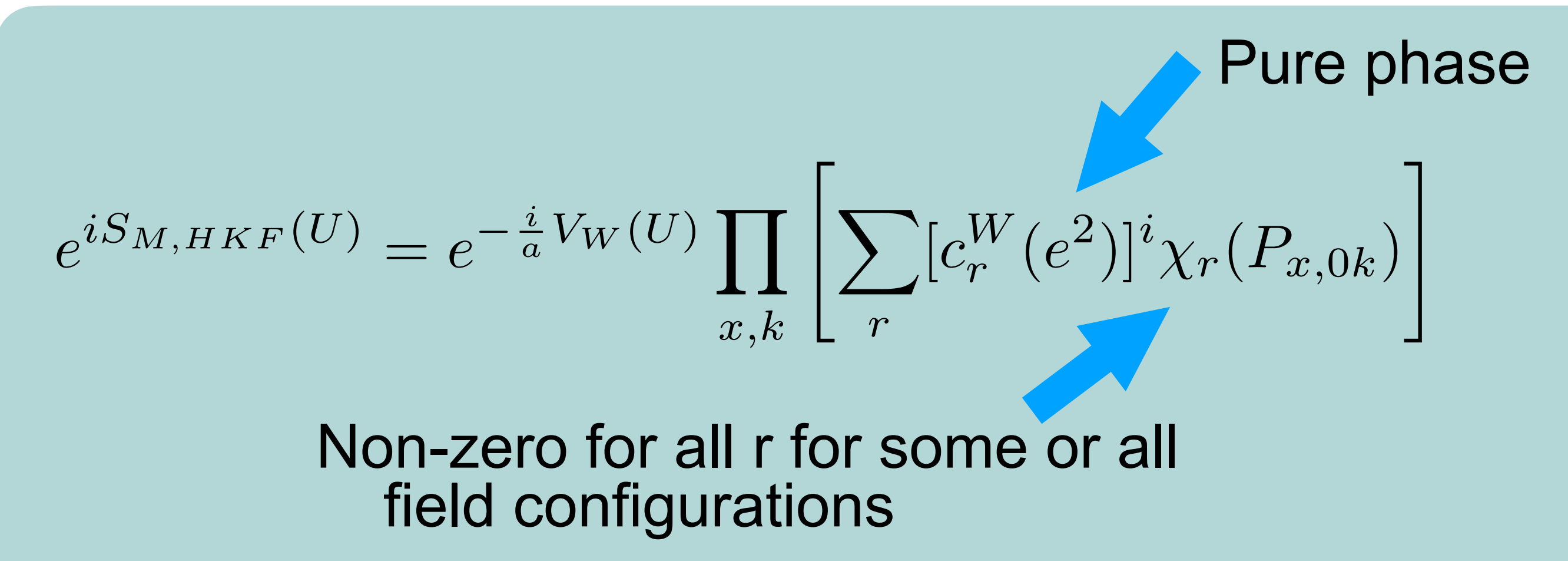

Rapid phase fluctuations lead to convergence of HFK path integrals, but without absolute convergence impossible to perform sum over representations using Monte Carlo methods 


\section{Changing paths}

Consider a path integral with a sign problem

$$
\langle\mathcal{O}\rangle_{M}=\frac{1}{Z_{M}} \int_{\mathcal{M}} \mathcal{D} U e^{i S_{M}(U)} \mathcal{O}(U)
$$

Many previous works:

Witten, AMS/IP

Stud.Adv.Math. 50 (2011)

Cristoforetti, Di Renzo,

Scorzato, PRD 86 (2012)

Deform the integration contour

$$
\begin{aligned}
& =\frac{1}{Z_{M}} \int_{\widetilde{\mathcal{M}}} \mathcal{D} U e^{i S_{M}(\widetilde{U})} \mathcal{O}(\widetilde{U}) \\
& =\frac{1}{Z_{M}} \int_{\mathcal{M}} \mathcal{D} U J(U) e^{i S_{M}(\widetilde{U}(U))} \mathcal{O}(\widetilde{U}(U))
\end{aligned}
$$
...

\section{Recent review:}

Alexandru, Basar, Bedaque, Warrington, arXiv:2007.05436

Deformed integrand can have less severe sign problem

$$
\begin{gathered}
=\frac{1}{Z_{M}} \int_{\mathcal{M}} \mathcal{D} U|J(U)| e^{-\operatorname{Im}\left[S_{M}(\widetilde{U}(U))\right]} \mathcal{O}(\widetilde{U}(U)) \\
\quad \times e^{i \operatorname{Re}\left[S_{M}(\widetilde{U}(U))\right]+i \arg [J(U)]}
\end{gathered}
$$




\section{A toy sign problem}

$$
\left\langle e^{i \phi}\right\rangle_{\beta}=\frac{1}{Z} \int_{-\pi}^{\pi} \frac{d \phi}{2 \pi} e^{i \phi} e^{\beta \cos (\phi)}=\frac{I_{1}(\beta)}{I_{0}(\beta)}
$$

Stokes' theorem + holomorphic integrand integral result unaffected by contour deormation

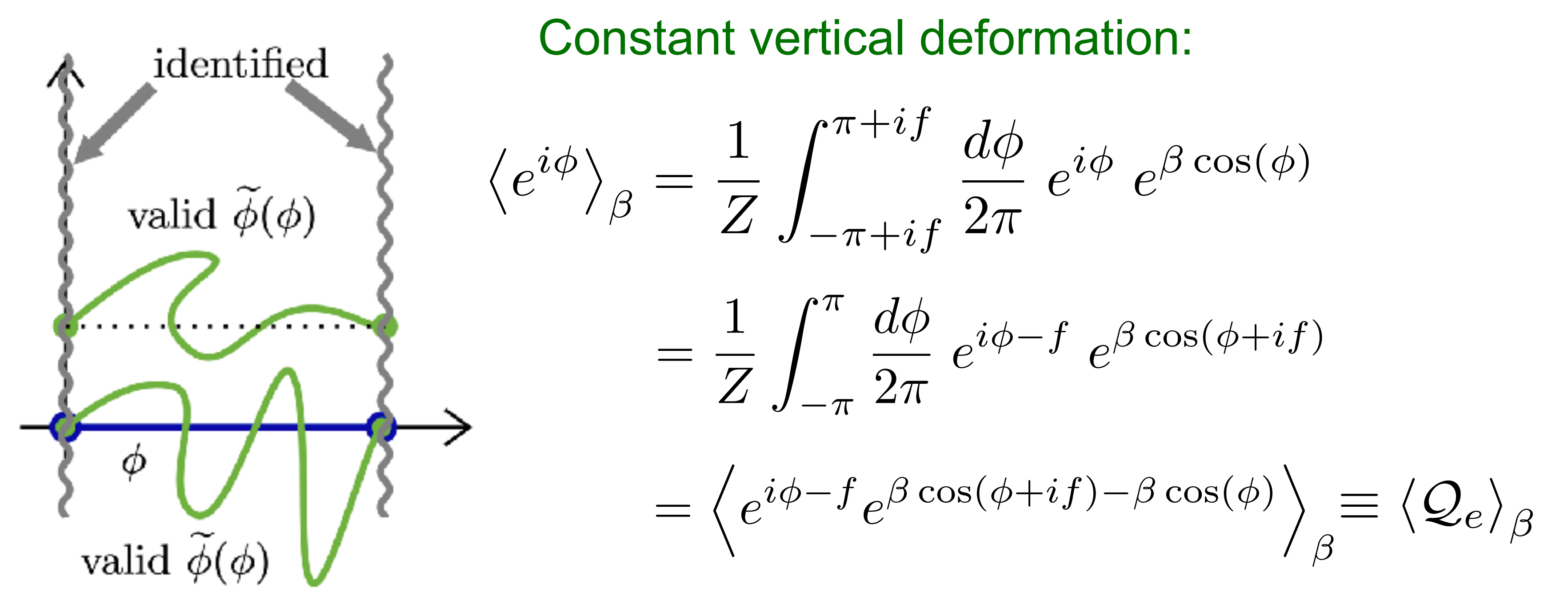




\section{Variance reduction}

The variance involves non-holomorphic integrands

$\operatorname{Var}_{\beta}\left[\operatorname{Re} \mathcal{Q}_{e}\right]=\left\langle\left(\operatorname{Re} \mathcal{Q}_{e}\right)^{2}\right\rangle_{\beta}-\left\langle e^{i \phi}\right\rangle_{\beta}^{2} \neq \operatorname{Var}_{\beta}\left[\operatorname{Re} e^{i \phi}\right]$

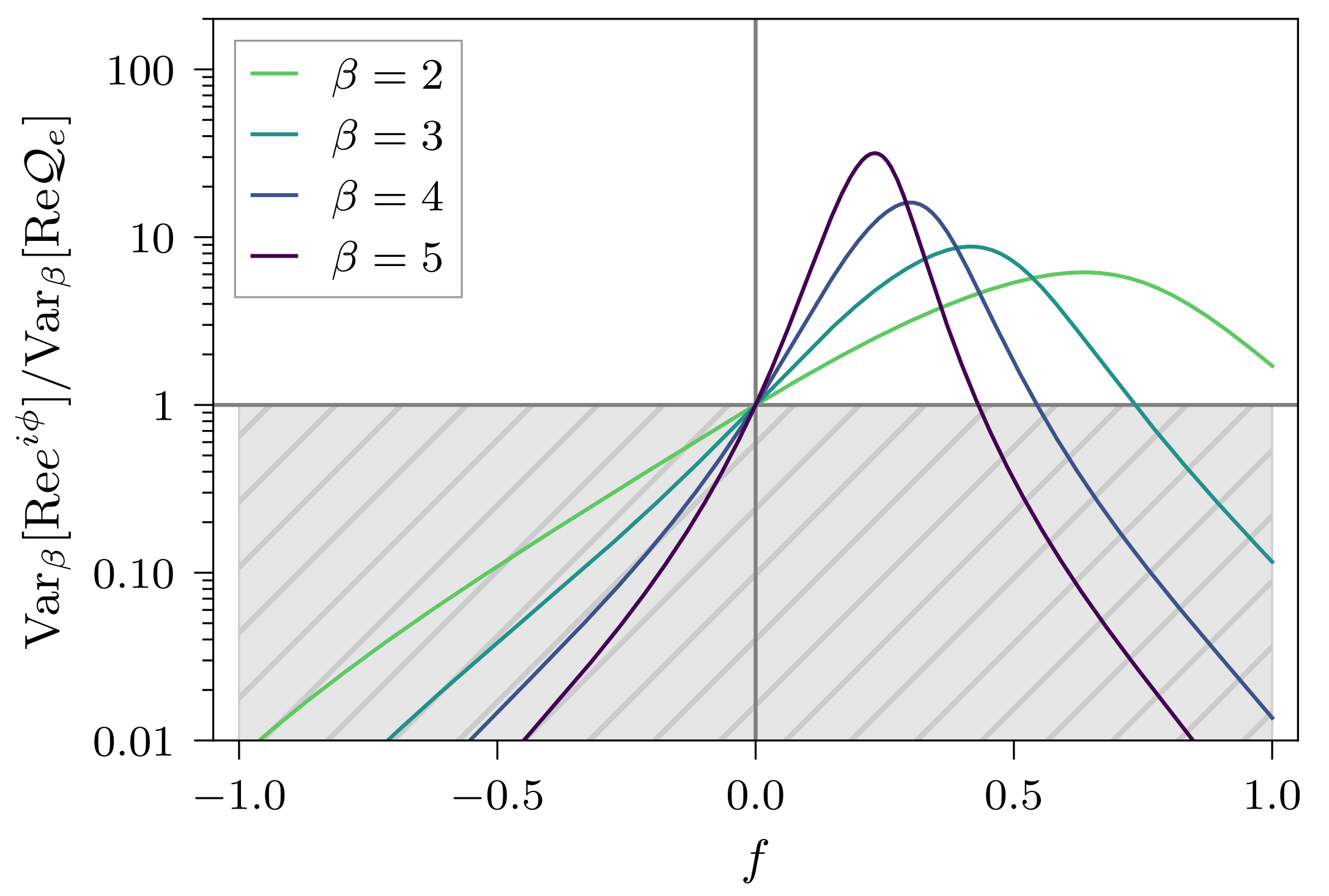

$\mathcal{Q}_{e}=e^{-f} e^{i \phi} e^{\Delta S}$ 


\section{Deformed observables}

Deformed observables method: contour deformations without modifying Monte Carlo sampling

$$
\begin{aligned}
& \langle\mathcal{O}\rangle=\frac{1}{Z} \int_{\mathcal{M}} \mathcal{D} U e^{-S(U)} \mathcal{O}(U) \\
& =\frac{1}{Z} \int_{\widetilde{\mathcal{M}}} \mathcal{D} \widetilde{U} e^{-S(\widetilde{U})} \mathcal{O}(\widetilde{U}) \\
& =\frac{1}{Z} \int_{\mathcal{M}} \mathcal{D} U e^{-S(U)} \operatorname{det}\left(\frac{\partial \widetilde{U}}{\partial U}\right) e^{-S(\widetilde{U}(U))+S(U)} \mathcal{O}(\widetilde{U}(U)) \\
& \equiv \frac{1}{Z} \int_{\mathcal{M}} \mathcal{D} U e^{-S(U)} \mathcal{Q}(U) \\
& \begin{array}{c}
\langle\mathcal{O}\rangle=\langle\mathcal{Q}\rangle \\
\operatorname{Var}[\mathcal{O}] \neq \operatorname{Var}[\mathcal{Q}]
\end{array} \\
& \begin{array}{c}
\langle\mathcal{O}\rangle=\langle\mathcal{Q}\rangle \\
\operatorname{Var}[\mathcal{O}] \neq \operatorname{Var}[\mathcal{Q}]
\end{array}
\end{aligned}
$$




\section{D U(1) contour deformations}

Using the parameterization

$$
P=e^{i \phi} \in U(1)
$$

$$
\left\langle W_{\mathcal{A}}\right\rangle=\left(\int \frac{d P}{2 \pi I_{0}\left(1 / e^{2}\right)} P e^{\frac{1}{2 e^{2}}\left(P+P^{-1}\right)}\right)^{A}
$$

$U(1)$ Wilson loops are products of toy sign problem integrals

$$
=\left(\int_{-\pi}^{\pi} \frac{d \phi}{2 \pi I_{0}\left(1 / e^{2}\right)} e^{i \phi} e^{\frac{1}{e^{2}} \cos (\phi)}\right)^{A}
$$

Contour deformation analogous to toy problem for $\mathrm{U}(1)$ Wilson loops

$$
e^{i \phi} \rightarrow e^{i(\phi+i f)}
$$
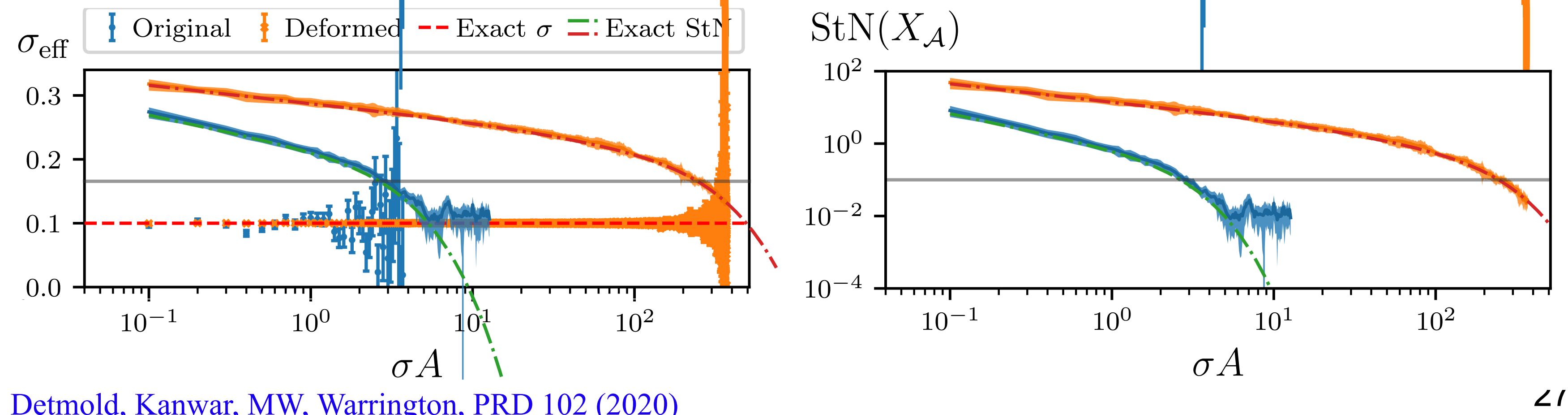

Detmold, Kanwar, MW, Warrington, PRD 102 (2020) 


\section{D SU(3) contour deformations}

Variance minimization of parameterized deformations is a well-posed optimization problem suitable for machine learning techniques

Parameterization and optimization strategies recently explored for $\mathrm{SU}(\mathrm{N})$
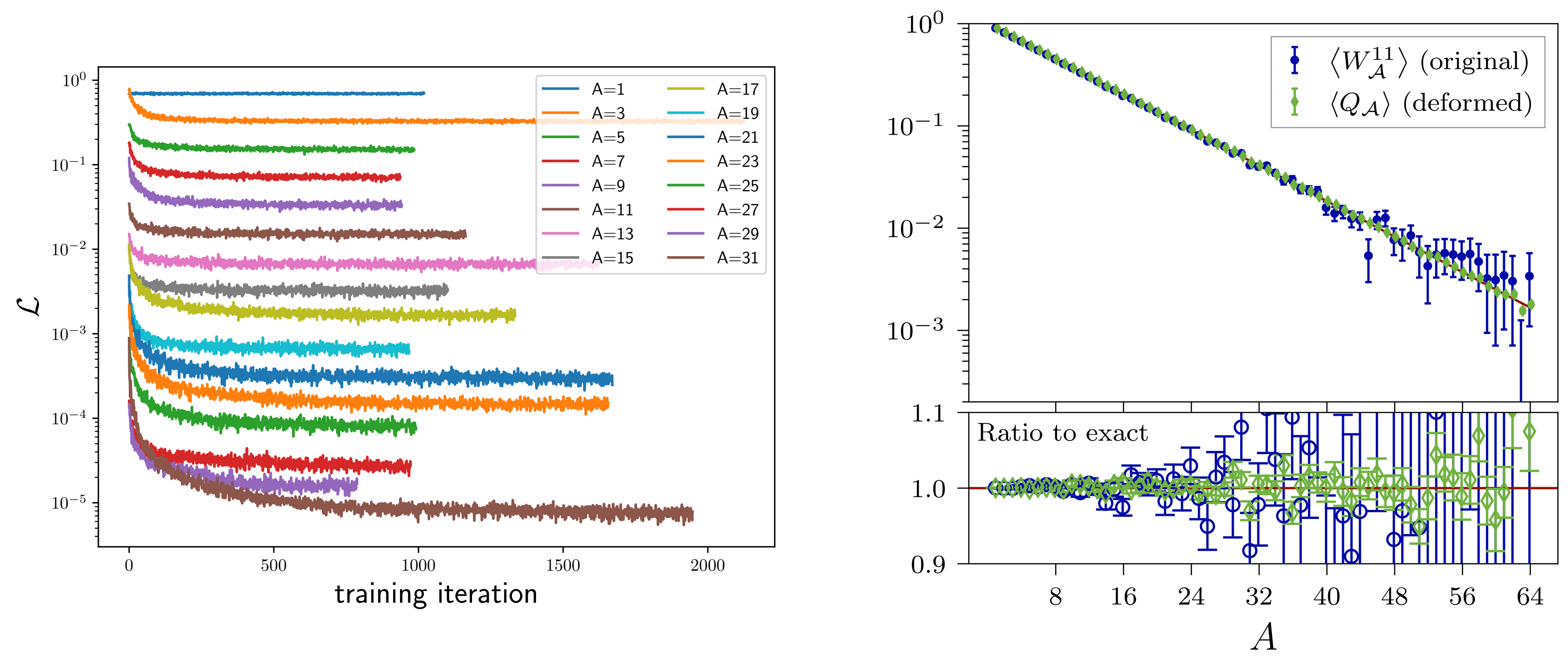


\section{Deformations and convergence}

Contour deformation methods can also improve convergence of real-time unitary actions

$$
\begin{aligned}
& \sum_{\{n\}} \int \mathcal{D} U e^{i S_{M}(U, n)}=\sum_{\{n\}} \int \mathcal{D} U J(U) e^{i S_{M}(\widetilde{U}(U, n), n)} \\
& \text { Convergent, but not } \\
& \text { absolutely } \\
& \text { (can't Monte Carlo) } \\
& e^{-\operatorname{Im}\left[S_{M}(\widetilde{U}(U, n), n)\right]}
\end{aligned}
$$

If (and only if) absolutely convergent path integral representation exists, can use Monte Carlo to perform joint sum-integral 


\section{Convergent U(1) HFK ?}

A simple contour deformation appears to provide convergence

$$
\begin{aligned}
& \phi_{x, 0 k} \rightarrow \widetilde{\phi}_{x, 0 k}=\phi_{x, 0 k}+i \operatorname{sign}\left(r_{x, k}\right) \\
& e^{i S_{M, H K F}(\widetilde{U}, r)}=e^{-\frac{i}{a} V_{W}(\widetilde{U})} \prod_{x, k}\left[\left[c_{r}^{W}\left(e^{2}\right)\right]^{i} e^{i r_{x, k} \phi_{x, 0 k}} e^{-\left|r_{x, k}\right|}\right] \\
& \widetilde{\phi}_{x, 0 k}=\phi_{x, 0 k}+i \alpha_{x, k} \\
& \alpha \in\left[0, \operatorname{sign}\left(r_{x, k}\right)\right]
\end{aligned}
$$

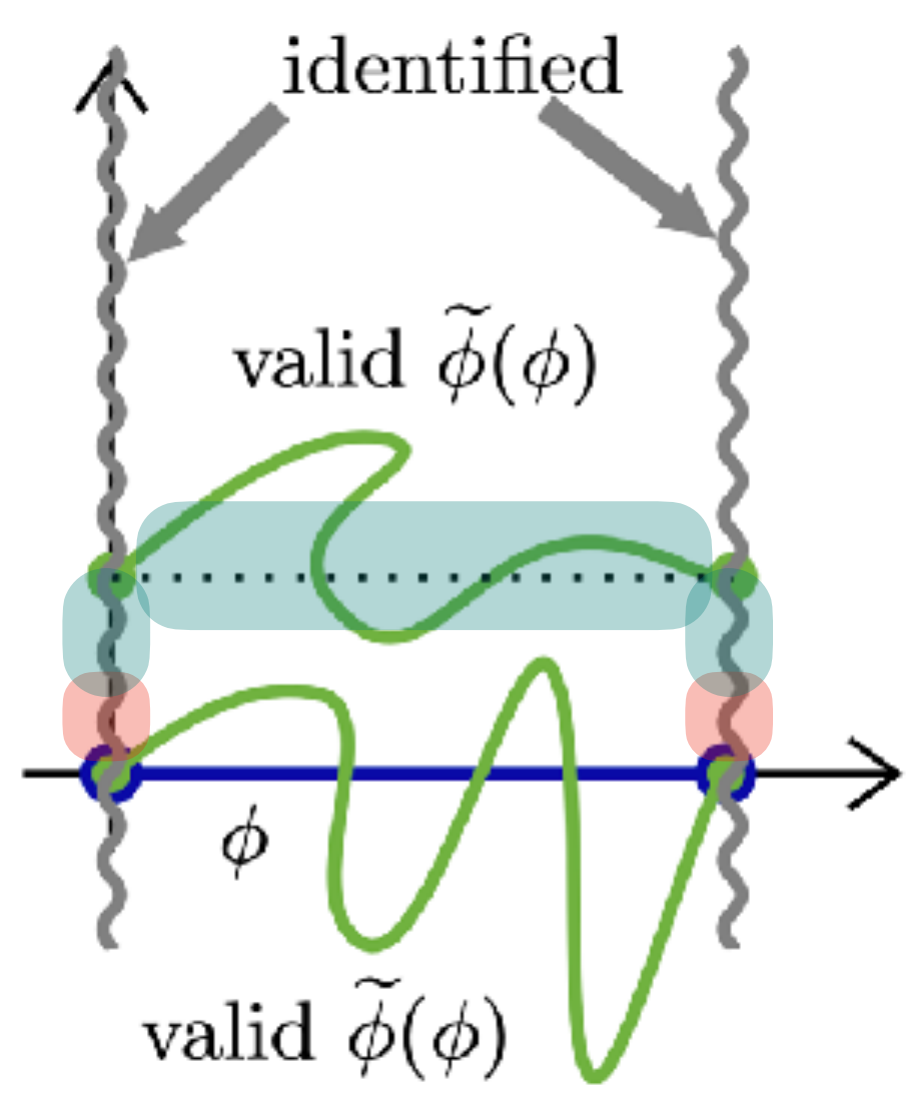




\section{Wick rotation regularization}

Minkowski action regularized by introducing "Wick rotation" angle

$$
\begin{gathered}
\theta \in[0, \pi / 2] \quad \begin{array}{c}
\text { Euclidean: } \quad \theta=0 \\
\text { Minkowski: } \quad \theta=\pi / 2
\end{array} \\
e^{i S_{M, H K F}(U)} \rightarrow e^{-\frac{i}{a} V_{W}(U)} \prod_{x, k}\left[\sum_{r=-\infty}^{\infty}\left[c_{r}^{W}\left(e^{2}\right)\right]^{e^{i \theta}} e^{i r \phi_{x, 0 k}}\right]
\end{gathered}
$$

Sum absolutely convergent for $\theta<\pi / 2$

Recipe for real-time path integrals

1) Regularize with Wick rotation angle

2) Perform contour deformation, enforcing cancellations arising from shift symmetry

3) Take Minkowski limit on deformed contour

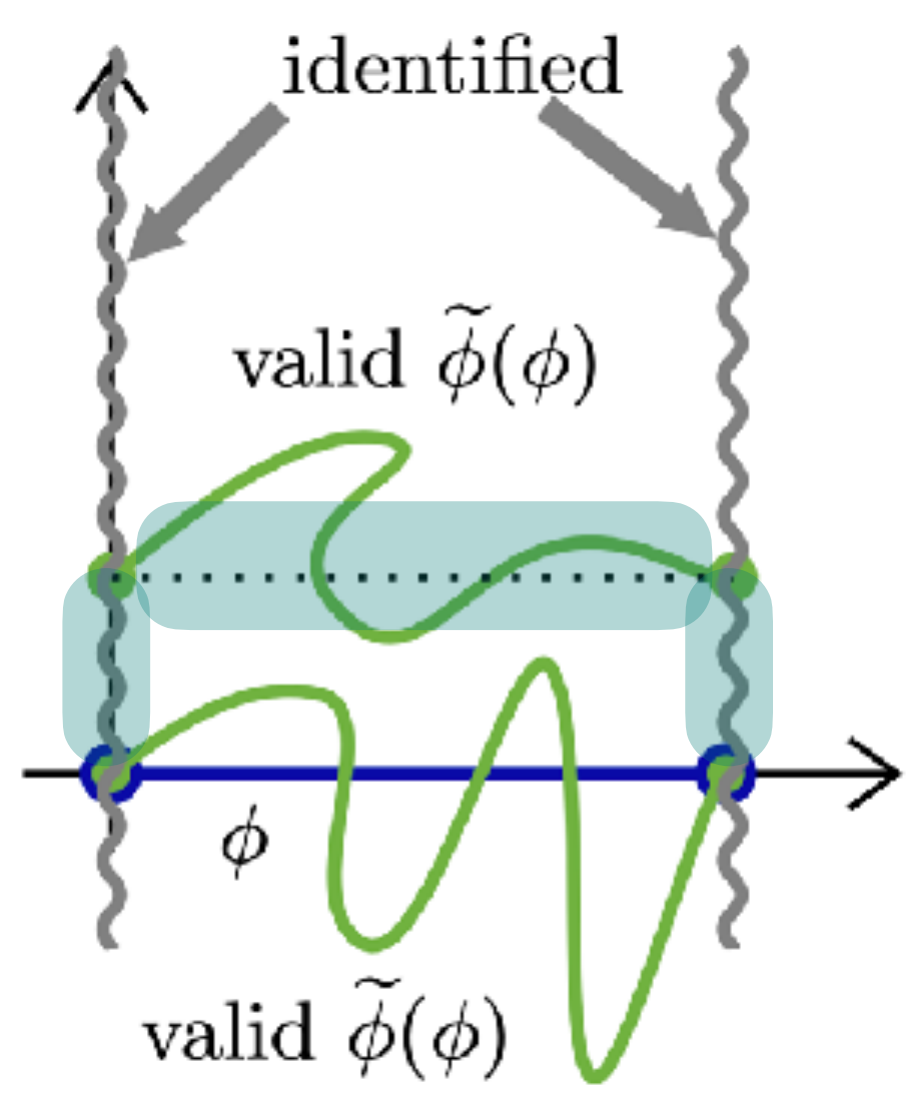




\section{Real-time U(1) HFK results}

Infinite sum in contour deformed real-time HFK action can be performed stochastically with integer-valued auxiliary field

$$
\int \mathcal{D} U J(U) e^{-S(\widetilde{U})}=\left(\int \mathcal{D} U \sum_{\{r\}}\right) e^{-S(\widetilde{U}, r)}
$$

Results consistent with exact $(1+1) \mathrm{D}$ analytic continuation
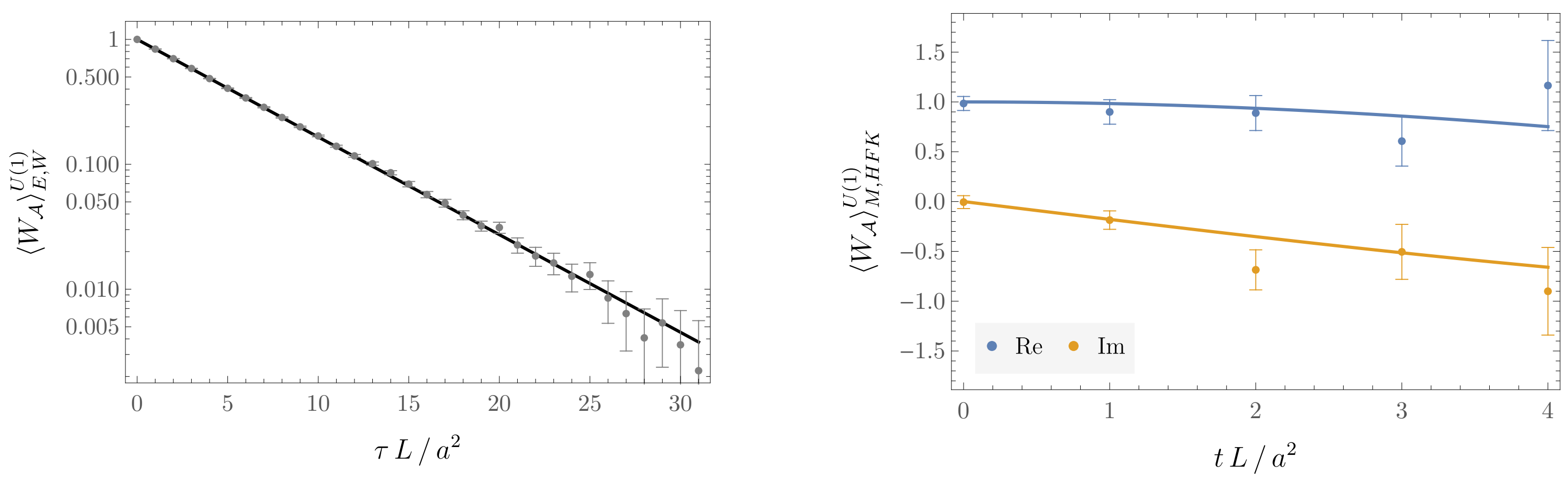

Real-time noisier, contour deform improves but doesn't completely remove sign problem 


\section{What about SU(N) ?}

Wick rotation of kinetic term still provides regularization

Sum more complicated, involves functions whose magnitudes can't be reduced using vertical deformations

$$
e^{i r \phi} \rightarrow \frac{\sin ((r+1) \phi)}{\sin (\phi)}
$$

Analogous definition of convergent HFK path integrals for lattice QCD possible, but we haven't found it 


\section{The heat-kernel equation}

Alternative starting point - Kogut-Susskind Hamiltonian

$$
\hat{H}=-\frac{g^{2}}{2 a} \sum_{x, k} \hat{\Delta}_{x, k}+V_{W}(\hat{U})
$$

Wilson action is in eigenbasis of potential

Eigenbasis of kinetic operator - solutions to "heat-kernel" equation

$$
\partial_{\tau} \mathcal{K}_{E}(U, \tau)=\Delta \mathcal{K}_{E}(U, \tau)
$$

Solution for $\mathrm{U}(1): \mathcal{K}_{E, U(1)}\left(e^{i \phi},-e^{2}\right)=\sum_{n=-\infty}^{\infty} \exp \left[-\frac{1}{2 e^{2}}(\phi+2 \pi n)^{2}\right]$

Generalization to SU(N) starting point for heat-kernel action 


\section{The heat-kernel action}

Solution for $\mathrm{SU}(\mathrm{N})$ :

Eigenvalue phases

$$
\mathcal{K}_{E, S U(N)}\left(U, \frac{g^{2}}{2}\right)=\sum_{n_{A}=-\infty}^{\infty} \mathcal{J}(\{\phi\},\{n\}) \exp \left[-\frac{1}{g^{2}}\left(\phi^{A}+2 \pi n^{A}\right)^{2}\right]
$$

Ugly but known, non-singular function

$$
\text { SU(N) constraint: } \quad \sum_{A=1}^{N} \phi^{A}=\sum_{A=1}^{N} n^{A}=0
$$

Isotropic Euclidean action with right naive continuum limit:

$$
e^{-S_{E, H K}(U)}=\prod_{x, \mu<\nu} \mathcal{K}_{E}\left(P_{x, \mu \nu}, \frac{g^{2}}{2}\right)
$$




\section{The Schrödinger equation}

Analytic continuation of heat-kernel equation gives Schrödinger equation on gauge group

$$
i \partial_{t} \mathcal{K}_{M}(U, t)=-\Delta \mathcal{K}_{M}(U, t)
$$

Euclidean solution can be analytically continued straightforwardly

$$
\begin{aligned}
& \mathcal{K}_{M, U(1)}\left(e^{i \phi}, e^{2}\right)=\sum_{n=-\infty}^{\infty} \exp \left[\frac{i}{2 e^{2}}(\phi+2 \pi n)^{2}\right] \\
& \mathcal{K}_{M, S U(N)}\left(U, \frac{g^{2}}{2}\right)=\sum_{n_{A}=-\infty}^{\infty} \mathcal{J}(\{\phi\},\{n\}) \exp \left[\frac{i}{g^{2}}\left(\phi^{A}+2 \pi n^{A}\right)^{2}\right]
\end{aligned}
$$




\section{More divergences}

Minkowski analog of heat-kernel action

$$
e^{i S_{M, H K}(U)}=\prod_{x, k} \mathcal{K}_{M}\left(P_{x, 0 k}, \frac{g^{2}}{2}\right) \prod_{x, i<j} \mathcal{K}_{M}\left(P_{x, \mu \nu},-\frac{g^{2}}{2}\right)
$$

Includes different but analogously divergent series

$$
\begin{aligned}
& \mathcal{K}_{M, U(1)}\left(e^{i \phi}, e^{2}\right)=\sum_{n=-\infty}^{\infty} \exp \left[\frac{i}{2 e^{2}}(\phi+2 \pi n)^{2}\right] \quad \begin{array}{c}
\text { Non-vanishising } \\
\text { for large n }
\end{array} \\
& \mathcal{K}_{M, S U(N)}\left(U, \frac{g^{2}}{2}\right)=\sum_{n_{A}=-\infty}^{\infty} \mathcal{J}(\{\phi\},\{n\}) \exp \left[\frac{i}{g^{2}}\left(\phi^{A}+2 \pi n^{A}\right)^{2}\right]
\end{aligned}
$$

Field configurations with infinitely many winding numbers all contribute to path integrals, suppressed by rapid phase fluctuations 


\section{The $\overline{\mathrm{HK}}$ action}

No symmetries lost by changing potential term

$$
e^{i S_{M, \overline{\mathrm{HK}}}(U)}=e^{-i a V_{W}(U)} \prod_{x, k} \mathcal{K}_{M}\left(P_{x, 0 k}, \frac{g^{2}}{2}\right)
$$

Divergence now only arises in kinetic term and takes the form of sum over Gaussian phases (times ugly but known function)

$$
\mathcal{K}_{M, S U(N)}\left(U, \frac{g^{2}}{2}\right)=\sum_{n_{A}=-\infty}^{\infty} \mathcal{J}(\{\phi\},\{n\}) \exp \left[\frac{i}{g^{2}}\left(\phi^{A}+2 \pi n^{A}\right)^{2}\right]
$$

Amenable to same strategy as U(1) HFK:

1) regularize kinetic term

2) deform integration contour to provide convergence

3) remove regulator 


\section{Convenient variables}

In order to perform contour deformations on eigenvalue phases, we need a few changes of variables

Temporal boundary conditions or Euclidean segments can be used to solve equations of motion for links in terms of plaquettes

$$
\left\{U_{x, \mu}, n_{x, k}^{A}\right\} \leftrightarrow\left\{P_{x, 0 k}, U_{x, 0}, n_{x, k}^{A}\right\}
$$

Eigenvector matrices $V_{x, 0 k}$ can be "integrated in" freely

$$
\begin{aligned}
\left\{U_{x, \mu}, n_{x, k}^{A}\right\} \leftrightarrow & \left\{\phi_{x, 0 k}^{A}, V_{x, 0 k}, U_{x, 0}, n_{x, k}^{A}\right\} \\
& A=1, \ldots, N
\end{aligned}
$$

Correlations from $\mathrm{SU}(\mathrm{N})$ constraint can be diagonalized

$$
\begin{aligned}
\left\{U_{x, \mu}, n_{x, k}^{A}\right\} \leftrightarrow & \left\{\psi_{x, 0 k}^{A}, V_{x, 0 k}, U_{x, 0}, m_{x, k}^{A}\right\} \\
& A=1, \ldots, N-1
\end{aligned}
$$




\section{Convergent SU(N) $\overline{\mathrm{HK}}$}

Wick rotated heat-kernel kinetic term in nice variables

$$
\mathcal{G}=\mathcal{J}(\{\phi\},\{n\}) \prod_{A=1}^{N-1} e^{\frac{i}{g^{2}} \rho^{A}\left(\psi^{A}+2 \pi m^{A}\right)^{2}}
$$

n-dependent contour deformation:
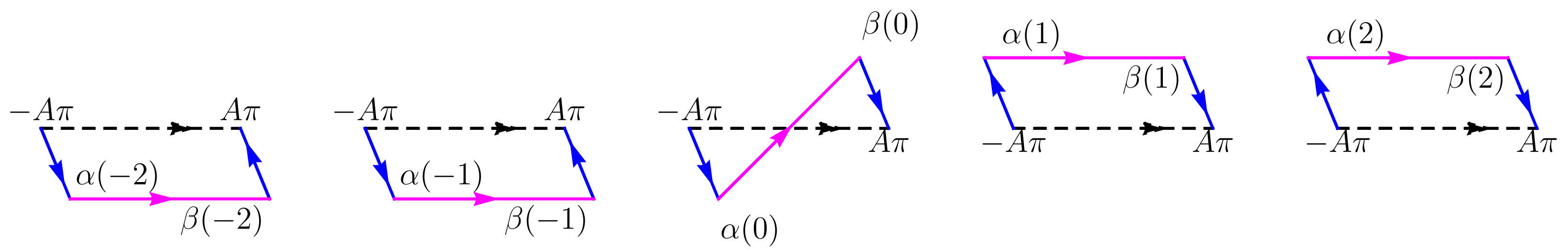

Provides exponential convergence everywhere except in neighborhood $\mathcal{G} \sim e^{-\mathcal{C}|n|}$ of endpoints 


\section{Convergent $\mathrm{SU}(\mathrm{N}) \overline{\mathrm{HK}}$}

Wick rotation provides absolutely convergence everywhere on deformed contour

$$
\mathcal{G} \rightarrow \mathcal{J}(\{\phi\},\{n\}) \prod_{A=1}^{N-1} e^{-\frac{1}{g^{2}} e^{-i \theta} \rho^{A}\left(\psi^{A}+2 \pi m^{A}\right)^{2}}
$$

Blue contours cancel by shift symmetry for all Wick rotation angle

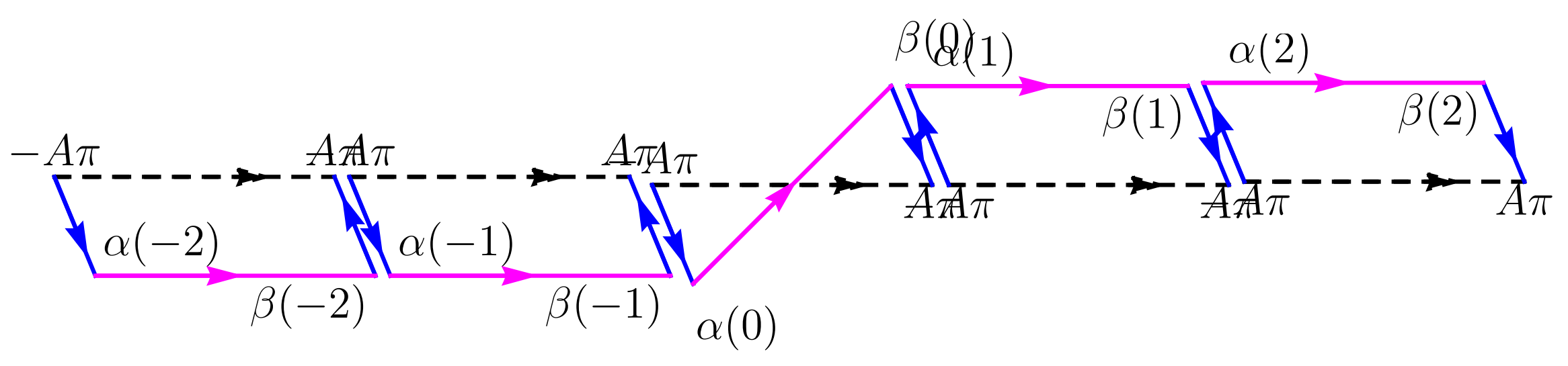

After enforcing cancellation of blue segments, sum-integral on pink contour is absolutely convergent for all gauge field values

Absolutely convergent $\mathrm{SU}(\mathrm{N})$ path integrals defined by taking Minkowski limit after cancelling blue contours 


\section{Real-time U(1) $\overline{\mathrm{HK}}$ results}

Similar stochastic sampling of auxiliary integer variables works for heat-kernel action

For $\mathrm{n}=0$ terms (dominant in classical approximation), this contour deform completely removes sign problem

$$
e^{\frac{i}{2 e^{2}} \phi^{2}} \rightarrow e^{-\frac{1}{2 e^{2}} \phi^{2}}
$$

Correspondingly no signal-to-noise degradation of $\left\langle e^{i \operatorname{Re}\left[S_{M}\right]}\right\rangle=1$
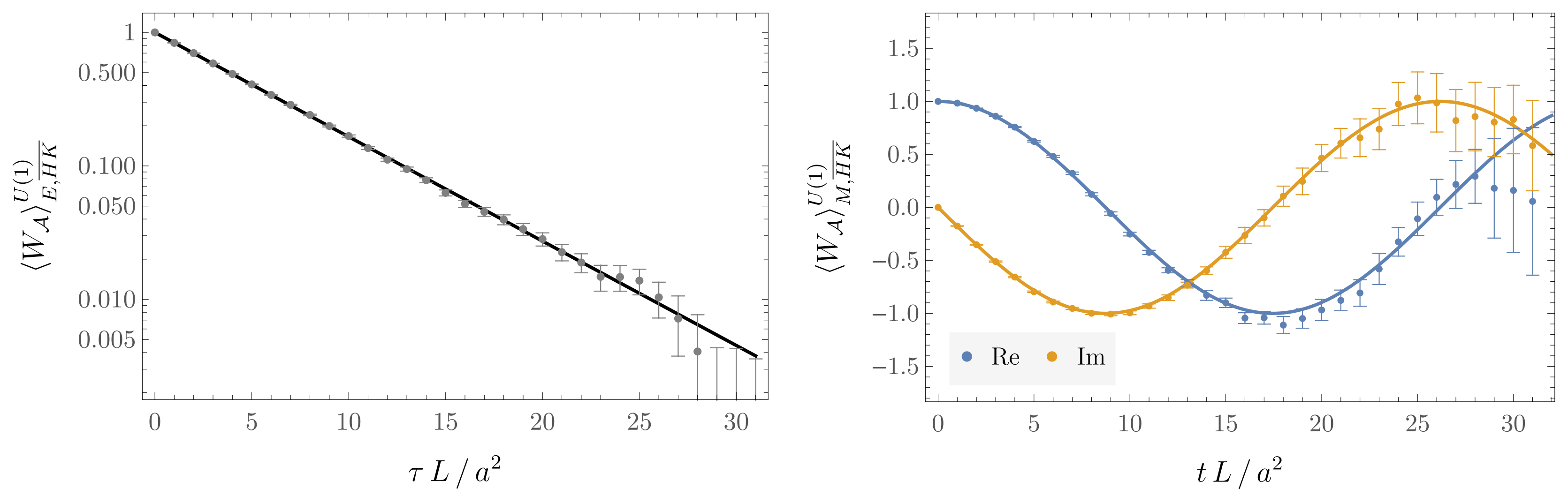


\section{Real-time $\mathrm{SU}(3) \overline{\mathrm{HK}}$ results}

Similar sampling strategies work for SU(3)

For $\mathrm{n}=0$ terms, contour deform similarly removes Gaussian phase fluctuations

Remaining phase fluctuations from Jacobian and heat-kernel prefactor, partition function sign problem observed to be mild
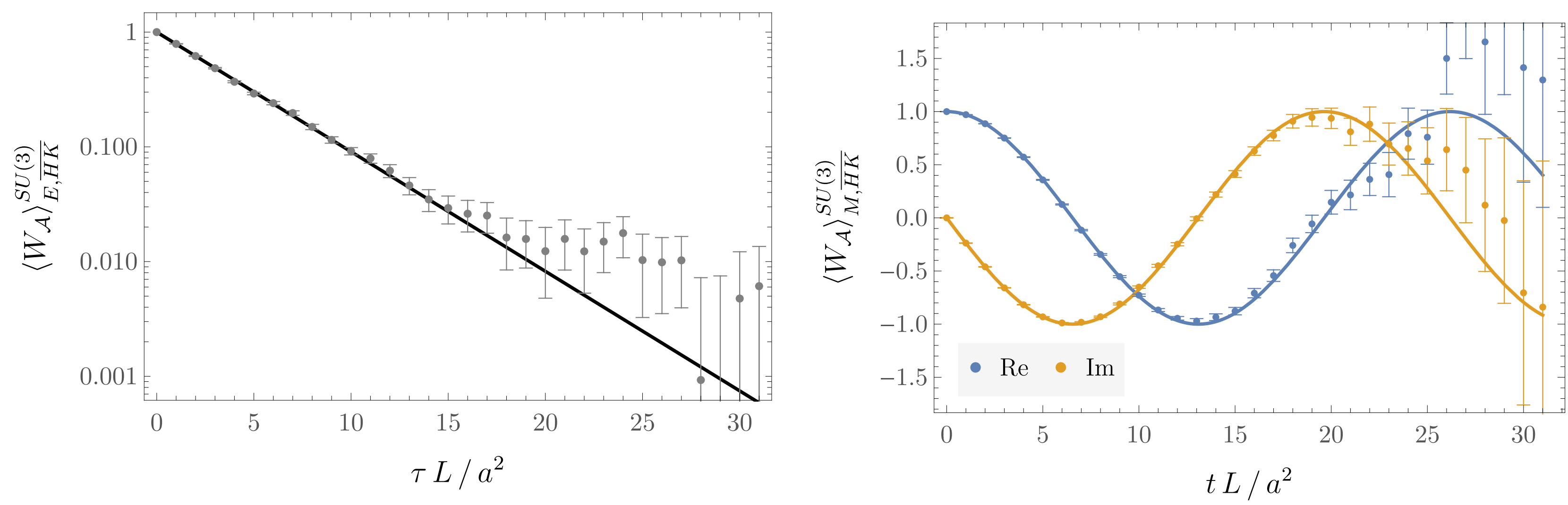


\section{Conclusions}

Many interesting questions about gauge theory involve challenges from sign problems
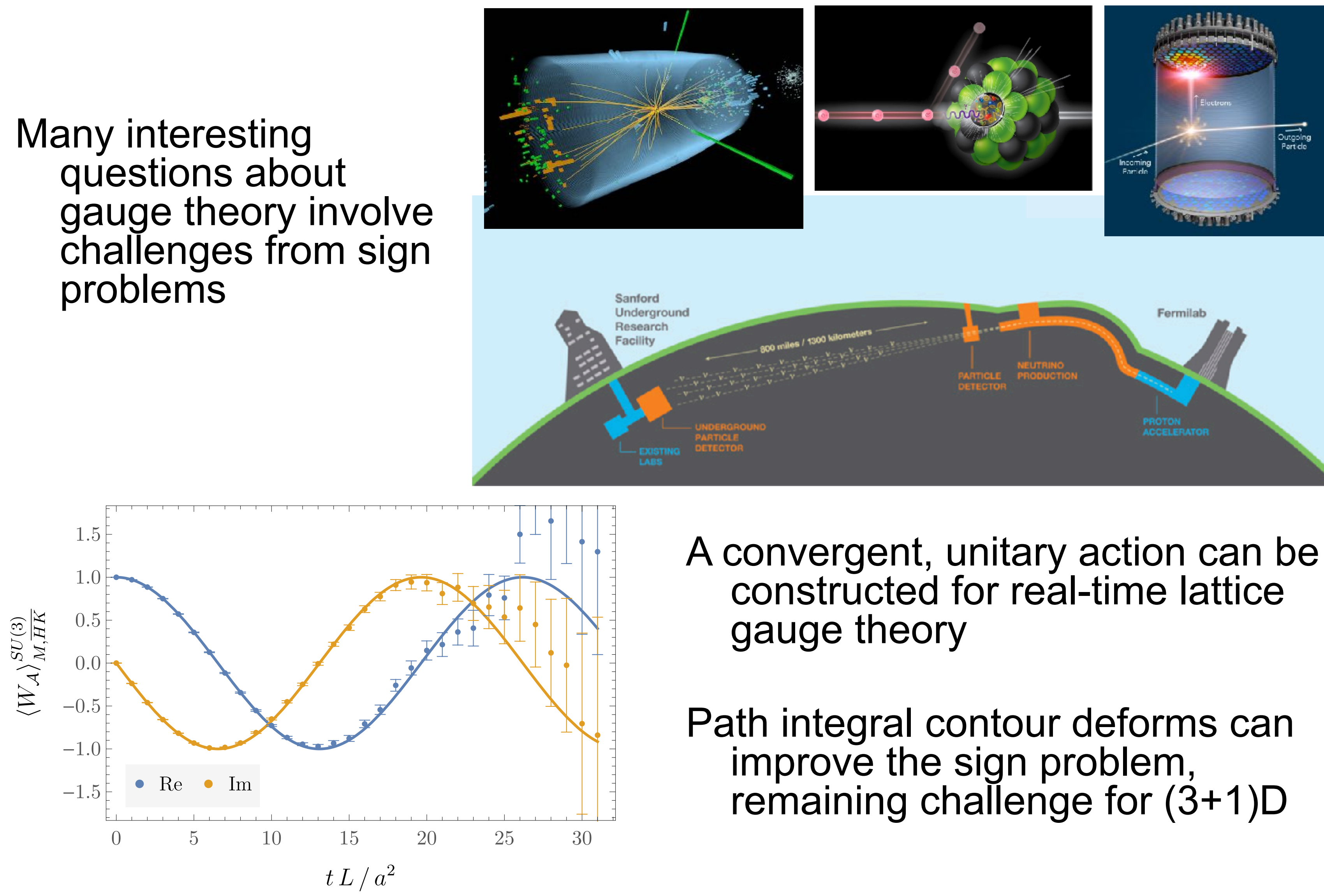

A convergent, unitary action can be constructed for real-time lattice gauge theory

Path integral contour deforms can improve the sign problem, remaining challenge for $(3+1) D$ 